\title{
Estado do conhecimento dos macroturbelários (Platyhelminthes) do Brasil
}

\author{
Fernando Carbayo ${ }^{1,3}$ \& Eudóxia Maria Froehlich ${ }^{2}$ \\ ${ }^{1}$ Escola de Artes, Ciências e Humanidades, Universidade de São Paulo - USP, \\ Av. Arlindo Bettio, 1000,CEP 03828-000,São Paulo,SP, Brasil,e-mail: baz@usp.br \\ ${ }^{2}$ Departamento de Zoologia, Instituto de Biociências, Universidade de São Paulo - USP, \\ Rua do Matão, Trav. 14, 321, Cidade Universitária, CEP 05508-900, São Paulo, SP, Brasil \\ ${ }^{3}$ Autor para correspondência: Fernando Carbayo,e-mail: baz@usp.br
}

CARBAYO, F. \& FROEHLICH, E.M. 2008. State of knowledge of the macroturbellarians (Platyhelminthes) from Brazil. Biota Neotrop. 8(4): http://www.biotaneotropica.org.br/v8n4/en/abstract?thematicreview+bn01908042008.

\begin{abstract}
In the last decades, international efforts have increased, with the aim of acquiring greater knowledge on worldwide biodiversity and to propose adequate conservation policies. Brazil has joined in these efforts, as is shown by recent publications on the state of knowledge of several taxa of organisms. The macroturbellarians (Polycladida and Tricladida, Platyhelminthes) are part of one of the less studied faunal groups, not only in Brazil but also in other regions of the world. In the present study we inventoried the knowledge on the diversity of Brazilian macroturbellarians and make a comparison with that from the rest of the world. We also analyze the accomplishments of Brazilian taxonomists dealing with macroturbellarians, with regard to the world context. Our results show that almost all of the 246 described species are from the South-eastern and Southern regions. The number of species will increase significantly when new samples are undertaken in biomes and regions still little or non-sampled, as well as in already explored areas. Brazil is relatively well provided with specialists in relation to other countries. Nonetheless, in view of the high numbers of estimated diversity, new samples should concur with the academic formation of new taxonomists.
\end{abstract}

Keywords: Turbellaria, Tricladida, Polycladida, biodiversity.

CARBAYO, F. \& FROEHLICH, E.M. 2008. Estado do conhecimento dos macroturbelários (Platyhelminthes) do Brasil. Biota Neotrop. 8(4): http://www.biotaneotropica.org.br/v8n4/pt/abstract?thematicreview+bn01908042008.

Resumo: Nas últimas décadas tem crescido o esforço internacional para conhecer a biodiversidade mundial visando a criação de políticas públicas de conservação. O Brasil vem participando deste esforço, como evidenciam as recentes publicações sobre o estado do conhecimento de vários táxons de organismos. Os macroturbelários (Polycladida e Tricladida, Platyhelminthes) fazem parte da fauna mundialmente menos estudada. Neste trabalho inventariamos por regiões a diversidade da fauna brasileira conhecida de macroturbelários e comparamos esta diversidade com a do resto do mundo. Analisamos também a atividade dos taxonomistas brasileiros do grupo no contexto mundial. Os resultados mostram que praticamente a totalidade das 246 espécies nominais descritas procede das regiões Sudeste e Sul. O número de espécies deverá aumentar significativamente quando forem feitas novas coletas, tanto em biomas e regiões ainda pouco ou nada explorados quanto nas áreas já amostradas. O Brasil está, relativamente a outros países, bem provido de especialistas. No entanto, em face da grande diversidade estimada, as amostragens deveriam ocorrer concomitantemente com a formação de novos especialistas.

Palavras-chave: Turbellaria, Tricladida, Polycladida, biodiversidade. 


\section{Introdução}

Os macroturbelários são os Platyhelminthes de vida livre de maiores dimensões, o que significa, no âmbito de "Turbellaria" (grupo parafilético, cf. Ehlers 1985), animais com mais de um milímetro de comprimento do corpo, embora o tamanho mais freqüente seja de 1-10 cm. Há dois grupos taxonômicos com estas características, as subordens Polycladida e Tricladida. Neste trabalho trataremos da subordem Polycladida como um todo e da subordem Tricladida considerando separadamente cada uma das suas quatro infraordens, i.e., Maricola, Cavernicola, Paludicola e Terricola. Com exceção de "Paludicola", grupo parafilético (Carranza et al. 1998), os outros quatro grupos são considerados monofiléticos.

Polycladida são turbelários que se caracterizam por apresentar ovos endolécitos e um ramo intestinal principal do qual se irradiam numerosos ramos (Hyman 1951). São exclusivamente marinhos (Prudhoe 1985).

Tricladida possuem ovos ectolécitos, intestino dividido em três ramos principais (Hyman 1951) e habitam ambientes aquáticos, límnicos ou marinhos, e ambientes terrestres. As quatro infraordens são classificadas de acordo com esses ambientes: Maricola, eminentemente em águas marinhas costeiras, com poucas espécies em água doce ou estuarinas (Sluys 1989); Cavernicola, em água doce de ambientes abertos ou de cavernas e no lençol freático (Sluys 1990); Paludicola, tipicamente de ambientes abertos de água doce, e Terricola, no meio terrestre úmido

Na recente avaliação do estado do conhecimento da biodiversidade brasileira (Lewinsohn 2006) para a elaboração de políticas futuras de manejo e conservação da biodiversidade, os turbelários, como os outros grupos, não foram tratados exaustivamente. No presente trabalho o nosso objetivo é complementar essa avaliação com informações mais completas sobre a diversidade dos macroturbelários.

\section{Material e Métodos}

Fizemos duas buscas bibliográficas. Na primeira, sobre a fauna brasileira, consultamos livros e trabalhos completos publicados em periódicos científicos: a) diretamente em nossos acervos bibliográficos; e b) nas bases de dados Biological Abstracts e Zoological Record (Thomson Reuters) (agosto 2008) com as palavras-chave Brazil e Turbellaria, e/ou Polycladida, e/ou Tricladida. Classificamos os trabalhos da primeira busca em 4 categorias: diversidade (descrições de espécies, sistemática, listas de espécies, chaves de identificação, faunística, filogenia, biogeografia); biologia e morfologia (ecologia, reprodução, regeneração, desenvolvimento, morfologia funcional, ultraestrutura, citogenética); biologia aplicada e técnicas (toxicidade, espécies bioindicadoras, técnicas de estudo) e estado do conhecimento. Usamos o termo registro para indicar a citação de uma espécie num município. Com auxílio da ferramenta de georreferenciamento Quantum GIS v.0.11.0 (http://www.qgis.org), criamos um mapa com indicação de todos os municípios em que há registro de macroturbelários, adotando para todos os municípios as coordenadas geográficas do IBGE disponíveis em http://splink.cria. org.br/geoloc. Não contabilizamos espécies não formalmente descritas. Para comparar a atividade dos sistematas de macroturbelários do Brasil com a dos especialistas de outros países, fizemos uma segunda busca dos trabalhos publicados nos últimos cinco anos, nas mesmas bases de dados (Biological Abstracts e Zoological Record, agosto 2008), com os termos Tricladida and Taxonomy, e Polycladida and Taxonomy. Dos trabalhos encontrados selecionamos aqueles em que novos táxons são descritos. Seguimos a classificação taxonômica de Tyler et al. (2006) para os "Turbellaria", e de Prudhoe (1985) para os Polycladida.

\section{Resultados e Discussão}

\section{Publicações}

Encontramos um total de 186 publicações sobre macroturbelários brasileiros, sendo 91 sobre diversidade, 66 sobre biologia e morfologia, 25 sobre biologia aplicada e técnicas, e 4 sobre o estado do conhecimento (Tabela 1). Estes quatro últimos trabalhos são sinópticos ou dedicados apenas às espécies do estado de São Paulo.

\section{Riqueza de espécies e endemismos de gêneros}

Existem 246 espécies (Tabela 2) (10,7\% do total mundial) e 64 gêneros (20,6\% do total mundial) de macroturbelários. Dezoito gêneros (5,8\% do total mundial) são endêmicos do Brasil (Tabela 3).

\section{Estado taxonômico das espécies}

No Brasil, o grupo mais rico em espécies conhecidas, Terricola, tem 24 espécies (15\%) com posição taxonômica duvidosa, pendente de reexame do material-tipo e/ou do estudo de exemplares adicionais. Todas estas espécies estão abrigadas no gênero coletivo Pseudogeoplana Ogren \& Kawakatsu 1990, criado justamente para espécies com dúvida. Algumas podem se revelar como sinônimos, outras como novas espécies, como já tem acontecido, especialmente, com as espécies de Graff (1899). Não identificamos problemas de identificação nos outros táxons, com exceção de um gênero monotípico de Maricola, Tiddles Marcus 1963, considerado incertae sedis (Sluys 1989).

\section{4. Áreas amostradas}

Conhecem-se macroturbelários de 13 estados brasileiros, abrangendo 121 municípios, os quais representam 2,2\% dos 5.560 municípios do país (IBGE 2001) (Figura 1). As regiões Sul e Sudeste incluem $88,4 \%$ dos municípios amostrados e $95,8 \%$ do total de registros (Tabela 4). Dos doze municípios litorâneos, dez são da região Sudeste (ES, RJ e SP) e dois da região Sul (PR).

\section{Estimativas de riqueza}

Estimativas sobre a riqueza de macroturbelários brasileiros não existem.

Polycladida. As 66 espécies deste grupo descritas -conhecidas de apenas 12 localidades (Figura 1) - devem representar uma pequena fração da riqueza real do grupo em face do relativamente pequeno esforço amostral. Até hoje, praticamente, só dois pesquisadores trabalharam com o grupo no Brasil, Ernst Marcus e Eveline du-Bois Reymond Marcus. Embora tivessem descrito mais de 60 espécies, novas para o Brasil ou novas para a ciência, as amostras foram basicamente manuais, em águas rasas e numa extensão de apenas $350 \mathrm{~km}$ da costa brasileira (du Bois-Reymond Marcus \& Marcus 1968), abrangendo somente uma das duas regiões biogeográficas oceânicas brasileiras (Spalding et al. 2007). Não foram ainda amostrados os ca. $3.000 \mathrm{~km}$ da costa brasileira de recifes, desde o Maranhão até o sul da Bahia (Maida \& Ferreira 1997), ecossistemas aos quais se atribui grande diversidade de Polycladida (Prudhoe 1985).

Tricladida, Maricola. No Brasil, as mesmas limitações das amostragens de Polycladida aplicam-se a este outro grupo marinho. No mundo, a distribuição geográfica das espécies do grupo é muito pouco conhecida. Sluys \& Kawakatsu (2005) identificaram várias áreas no planeta com riqueza de espécies relativamente alta, indicando o extremo sul da América do Sul como a de maior riqueza de espécies, e a costa do estado de São Paulo como a de maior número de endemismos. Esta última é praticamente a única região amostrada, no que diz respeito aos Maricola, de toda a costa brasileira. Considerando a 
Tabela 1. Publicações, em ordem alfabética de autor, sobre macroturbelários brasileiros, com indicação do assunto e do táxon principais abordados. div: diversidade; bio: biologia e morfologia; tec: biologia aplicada e técnicas; con: estado do conhecimento.

Table 1. Publications, alphabetically ordered by author, dealing with Brazilian macroturbellarians. For each publication, the main subject and taxon is also indicated. div: diversity; bio: biology and morphology; tec: applied biology and technics; con: state of knowledge.

\begin{tabular}{|c|c|c|}
\hline Referência & Assunto & $\begin{array}{c}\text { Táxon } \\
\text { principal }\end{array}$ \\
\hline Almeida et al. (1988) & bio & Terricola \\
\hline Almeida et al. (1991) & bio & Terricola \\
\hline Alvarez \& Almeida (1999) & bio & Terricola \\
\hline Alvarez \& Almeida (2002) & bio & Terricola \\
\hline Alvarez \& Almeida (2007) & bio & Terricola \\
\hline Anônimo (1987) & div & Maricola \\
\hline Antunes et al. (2008) & $\operatorname{div}$ & Terricola \\
\hline Ball (1969) & div & Paludicola \\
\hline Ball (1971) & div & Paludicola \\
\hline Baptista \& Leal-Zanchet (2005) & $\operatorname{div}$ & Terricola \\
\hline Baptista et al. (2006) & div & Terricola \\
\hline Barros et al. (2006) & tec & Paludicola \\
\hline Bell (1900) & div & Terricola \\
\hline Benya et al. (2007) & bio & Paludicola \\
\hline Blainville (1826) & $\operatorname{div}$ & Terricola \\
\hline Böhmig (1887) & div & Paludicola \\
\hline Borelli (1898) & div & Paludicola \\
\hline Bueno-Silva \& Fischer (2005a) & bio & Paludicola \\
\hline Bueno-Silva \& Fischer (2005b) & tec & Paludicola \\
\hline Bueno-Silva \& Fischer (2007) & bio & Paludicola \\
\hline Campos-Velho et al., 2004) & bio & Paludicola \\
\hline Carbayo (2005) & tec & Terricola \\
\hline Carbayo (2006) & $\operatorname{div}$ & Terricola \\
\hline Carbayo \& Leal-Zanchet (2001) & $\operatorname{div}$ & Terricola \\
\hline Carbayo et al. (2001) & tec & Terricola \\
\hline Carbayo et al. (2002) & tec & Terricola \\
\hline Carbayo \& Leal-Zanchet (2003) & div & Terricola \\
\hline Carbayo et al. (2008) & bio & Terricola \\
\hline Carlé (1935) & bio & Terricola \\
\hline Carvalho et al. (1975) & tec & Terricola \\
\hline Carvalho et al. (1984a) & bio & Terricola \\
\hline Carvalho et al. (1984b) & bio & Terricola \\
\hline Castro \& Leal-Zanchet (2005) & $\operatorname{div}$ & Terricola \\
\hline Corrêa (1947) & $\operatorname{div}$ & Terricola \\
\hline Corrêa (1949) & div & Polycladida \\
\hline Corrêa (1958) & div & Polycladida \\
\hline Corrêa (1964) & con & Maricola \\
\hline Darwin (1844) & $\operatorname{div}$ & Terricola \\
\hline Diesing (1861) & div & Terricola \\
\hline du Bois-Reymond Marcus (1951a) & bio & Turbellaria \\
\hline du Bois-Reymond Marcus (1951b) & $\operatorname{div}$ & Terricola \\
\hline du Bois-Reymond Marcus (1955a) & $\operatorname{div}$ & Polycladida \\
\hline du Bois-Reymond Marcus (1955b) & div & $\begin{array}{l}\text { Maricola, } \\
\text { Terricola }\end{array}$ \\
\hline
\end{tabular}

Tabela 1. Continuação...

\begin{tabular}{|c|c|c|}
\hline Referência & Assunto & $\begin{array}{c}\text { Táxon } \\
\text { principal }\end{array}$ \\
\hline du Bois-Reymond Marcus (1957) & $\operatorname{div}$ & Polycladida \\
\hline du Bois-Reymond Marcus (1958) & $\operatorname{div}$ & Polycladida \\
\hline du Bois-Reymond Marcus (1965) & $\operatorname{div}$ & Polycladida \\
\hline $\begin{array}{l}\text { du Bois-Reymond Marcus \& Marcus } \\
\text { (1968) }\end{array}$ & $\operatorname{div}$ & Polycladida \\
\hline Falleni et al. (2006) & bio & Terricola \\
\hline Faubel (1983) & $\operatorname{div}$ & Polycladida \\
\hline Faubel (1984) & $\operatorname{div}$ & Polycladida \\
\hline Fernandes et al. (2003) & bio & Terricola \\
\hline Férussac (1821) & $\operatorname{div}$ & Terricola \\
\hline Fick et al. (2006) & $\operatorname{div}$ & Terricola \\
\hline Fiorentin \& Friedrich (1985) & bio & Paludicola \\
\hline Forneris (1999) & con & Turbellaria \\
\hline Froehlich (1955a) & div & Terricola \\
\hline Froehlich (1955b) & $\operatorname{div}$ & Terricola \\
\hline Froehlich (1955c) & bio & Terricola \\
\hline Froehlich (1956a) & $\operatorname{div}$ & Terricola \\
\hline Froehlich (1956b) & $\operatorname{div}$ & Terricola \\
\hline Froehlich (1958) & $\operatorname{div}$ & Terricola \\
\hline Froehlich (1959) & $\operatorname{div}$ & Terricola \\
\hline Froehlich (1964) & con & Paludicola \\
\hline Froehlich (1966) & bio & Terricola \\
\hline Froehlich (1967) & $\operatorname{div}$ & Terricola \\
\hline E.M. Froehlich (1955a) & $\operatorname{div}$ & Terricola \\
\hline E.M. Froehlich (1955b) & $\operatorname{div}$ & Terricola \\
\hline E.M. Froehlich (1985) & tec & Terricola \\
\hline E.M. Froehlich \& Froehlich (1972) & $\operatorname{div}$ & Terricola \\
\hline E.M. Froehlich \& Leal-Zanchet (2003) & $\operatorname{div}$ & Terricola \\
\hline Goeldi (1894) & $\operatorname{div}$ & Terricola \\
\hline Graff (1896) & $\operatorname{div}$ & Terricola \\
\hline Graff (1899) & $\operatorname{div}$ & Terricola \\
\hline Guecheva et al. (2001) & tec & Paludicola \\
\hline Guecheva et al. (2003) & tec & Paludicola \\
\hline Guimarães \& Hirano (1971) & bio & Paludicola \\
\hline Guimarães \& Hirano (1973) & bio & Paludicola \\
\hline Guimarães et al. (1975) & bio & Paludicola \\
\hline Hauser (1959a) & bio & Terricola \\
\hline Hauser (1959b) & bio & Terricola \\
\hline Hauser (1979) & bio & Paludicola \\
\hline Hauser (1985) & bio & Paludicola \\
\hline Hauser (1987) & bio & Paludicola \\
\hline Hauser et al. (1979a) & tec & Paludicola \\
\hline Hauser et al. (1979b) & bio & Paludicola \\
\hline Hauser et al. (1979c) & bio & Paludicola \\
\hline Hauser et al. (1980) & bio & Paludicola \\
\hline Hauser \& Friedrich (1982) & bio & Paludicola \\
\hline Hauser \& Heller (1983) & bio & Paludicola \\
\hline Hauser \& Santos (1985a) & bio & Paludicola \\
\hline Hauser \& Santos (1985b) & bio & Paludicola \\
\hline Hauser \& Santos (1985c) & bio & Paludicola \\
\hline Heller (1985a) & bio & Paludicola \\
\hline
\end{tabular}


Tabela 1. Continuação...

\begin{tabular}{|c|c|c|}
\hline Referência & Assunto & $\begin{array}{c}\text { Táxon } \\
\text { principal }\end{array}$ \\
\hline Heller (1985b) & bio & Paludicola \\
\hline Hensel (1980) & bio & Paludicola \\
\hline Hyman (1955) & $\operatorname{div}$ & $\begin{array}{l}\text { Polycladida, } \\
\text { Maricola, } \\
\text { Terricola }\end{array}$ \\
\hline Jung et al. (1981) & bio & Paludicola \\
\hline Kawakatsu (1989) & $\operatorname{div}$ & Paludicola \\
\hline Kawakatsu et al. (1976) & $\operatorname{div}$ & Paludicola \\
\hline Kawakatsu et al. (1980) & $\operatorname{div}$ & Paludicola \\
\hline Kawakatsu et al. (1981) & div & Paludicola \\
\hline Kawakatsu et al. (1982) & $\operatorname{div}$ & Paludicola \\
\hline Kawakatsu et al. (1983a) & $\operatorname{div}$ & Paludicola \\
\hline Kawakatsu et al. (1983b) & $\operatorname{div}$ & Paludicola \\
\hline Kawakatsu et al. (1984) & $\operatorname{div}$ & Paludicola \\
\hline Kawakatsu et al. (1985) & $\operatorname{div}$ & Paludicola \\
\hline Kawakatsu et al. (1986) & $\operatorname{div}$ & Paludicola \\
\hline Kawakatsu \& E.M. Froehlich (1992) & $\operatorname{div}$ & Cavernicola \\
\hline Kawakatsu et al. (1992) & $\operatorname{div}$ & Paludicola \\
\hline Kenk (1974) & $\operatorname{div}$ & Paludicola \\
\hline Knakievicz \& Bunselmeyer (2008) & tec & Paludicola \\
\hline Knakievicz et al. (2006) & bio & Paludicola \\
\hline Knakievicz et al. (2007) & $\operatorname{div}$ & Paludicola \\
\hline Lacerda et al. (2005) & tec & Paludicola \\
\hline Lau et al. (2007) & tec & Paludicola \\
\hline Leal-Zanchet \& Hauser (1999) & bio & Paludicola \\
\hline Leal-Zanchet \& Carbayo (2000) & $\operatorname{div}$ & Terricola \\
\hline Leal-Zanchet \& Carbayo (2001) & $\operatorname{div}$ & Terricola \\
\hline Leal-Zanchet \& E.M. Froehlich (2001) & $\operatorname{div}$ & Terricola \\
\hline Leal-Zanchet \& E.M. Froehlich (2006) & div & Terricola \\
\hline Leal-Zanchet \& Souza (2003) & $\operatorname{div}$ & Terricola \\
\hline Leal-Zanchet \& Baptista (2008) & $\operatorname{div}$ & Terricola \\
\hline Leuck \& Luz (1980) & bio & Paludicola \\
\hline Lima (1981) & tec & Paludicola \\
\hline Lima (1982) & tec & Paludicola \\
\hline Lima (1984) & tec & Paludicola \\
\hline Marcus (1946) & $\operatorname{div}$ & Paludicola \\
\hline Marcus (1947) & div & $\begin{array}{l}\text { Polycladida, } \\
\text { Maricola }\end{array}$ \\
\hline Marcus (1948) & div & $\begin{array}{l}\text { Maricola, } \\
\text { Paludicola, } \\
\text { Polycladida }\end{array}$ \\
\hline Marcus (1949) & div & Polycladida \\
\hline Marcus (1950) & $\operatorname{div}$ & Polycladida \\
\hline Marcus (1951) & div & Terricola \\
\hline Marcus (1952) & $\operatorname{div}$ & $\begin{array}{l}\text { Polycladida, } \\
\text { Terricola }\end{array}$ \\
\hline Marcus (1954) & div & Polycladida \\
\hline Marcus (1963) & div & Maricola \\
\hline Martins (1970) & $\operatorname{div}$ & Paludicola \\
\hline Melo et al. (1995) & tec & Paludicola \\
\hline
\end{tabular}

Tabela 1. Continuação...

\begin{tabular}{|c|c|c|}
\hline Referência & Assunto & $\begin{array}{c}\text { Táxon } \\
\text { principal }\end{array}$ \\
\hline Melo \& Andrade (2001) & tec & Paludicola \\
\hline Moseley (1877) & $\operatorname{div}$ & Terricola \\
\hline Ogren \& Kawakatsu (1990) & $\operatorname{div}$ & Terricola \\
\hline Ogren et al. (1997) & $\operatorname{div}$ & Terricola \\
\hline Ogren \& Kawakatsu (1998) & $\operatorname{div}$ & Terricola \\
\hline Palma (1984) & bio & Paludicola \\
\hline Pereira (1970) & tec & Tricladida \\
\hline Prá et al. (2005) & tec & Paludicola \\
\hline Preza \& Smith (2001) & tec & Paludicola \\
\hline Prudhoe (1985) & $\operatorname{div}$ & Polycladida \\
\hline Riester (1938) & $\operatorname{div}$ & Terricola \\
\hline Rodrigues \& E.M. Froehlich (1998) & con & Turbellaria \\
\hline Santos \& Hauser (1984) & bio & Paludicola \\
\hline Sawaya \& Ungareti (1948) & bio & Paludicola \\
\hline Schirch (1929) & $\operatorname{div}$ & Terricola \\
\hline Schultze \& Müller (1857) & $\operatorname{div}$ & Terricola \\
\hline Schultze (1857) & $\operatorname{div}$ & Terricola \\
\hline Seitenfus \& Leal-Zanchet (2004) & tec & Terricola \\
\hline Silva et al. (1997) & tec & Paludicola \\
\hline Silveira (1969) & bio & Paludicola \\
\hline Silveira (1970) & bio & Paludicola \\
\hline Silveira (1973) & bio & Paludicola \\
\hline Silveira (1974) & bio & Paludicola \\
\hline Silveira (1998) & bio & Terricola \\
\hline Silveira \& Corinna (1976) & bio & Terricola \\
\hline Sluys (1989) & $\operatorname{div}$ & Maricola \\
\hline Sluys (1990) & $\operatorname{div}$ & Cavernicola \\
\hline Sluys (1996) & $\operatorname{div}$ & Paludicola \\
\hline Sluys (1999) & tec & Terricola \\
\hline Sluys \& Kawakatsu (2005) & $\operatorname{div}$ & Maricola \\
\hline Sluys et al. (1997) & $\operatorname{div}$ & Paludicola \\
\hline Sluys et al. (2005) & $\operatorname{div}$ & Paludicola \\
\hline Smith (1960) & div & Polycladida \\
\hline Souza (1954) & bio & Paludicola \\
\hline Souza \& Hauser (1984) & bio & Paludicola \\
\hline Souza \& Leal-Zanchet (2002) & bio & Paludicola \\
\hline Souza \& Leal-Zanchet (2004) & bio & Terricola \\
\hline Souza et al. (2005) & bio & Paludicola \\
\hline Steigleder \& Hauser (1984) & bio & Paludicola \\
\hline Valer (2001) & bio & Paludicola \\
\hline Vara et al. (2001) & tec & Paludicola \\
\hline Vara \& Leal-Zanchet (2008) & bio & Paludicola \\
\hline Veyl et al. (2002) & bio & Paludicola \\
\hline Wiilland et al. (1998) & bio & Paludicola \\
\hline Wiilland et al. (2004) & bio & Paludicola \\
\hline Wirth \& Heller (1985a) & bio & Paludicola \\
\hline Wirth \& Heller (1985b) & bio & Paludicola \\
\hline Wirth \& Heller (1985c) & bio & Paludicola \\
\hline Wirth \& Heller (1985d) & bio & Paludicola \\
\hline
\end{tabular}


Tabela 2. Espécies de macroturbelários (Polycladida e Tricladida) e município de ocorrência no Brasil. Os nomes dos municípios foram atualizados. Com o asterisco * foram marcadas localidades não representadas na Figura 1.

Table 2. Macroturbellarian species (Polycladida and Tricladida) and municipalities from where each species is known. The names of the municipalities are the presently used. The asterisk * indicates sites not plotted on the Figure 1.

\begin{tabular}{|c|c|c|}
\hline Ordem & Espécie & Município/Estado \\
\hline Polycladida & Acerotisa bituna Marcus 1947 & Guarujá/SP (Marcus 1947) \\
\hline Polycladida & Acerotisa leuca Marcus 1947 & Guarujá/SP (Marcus 1947) \\
\hline Polycladida & Adenoplana evelinae Marcus 1950 & Ilhabela/SP (Marcus 1950) \\
\hline Polycladida & Alleena callizona Marcus 1947 & Guarujá/SP (Marcus 1947) \\
\hline Polycladida & Alloioplana aulica (Marcus 1947) & Guarujá/SP (Marcus 1947) \\
\hline Polycladida & Armatoplana leptalea (Marcus 1947) & Guarujá/SP (Marcus 1947) \\
\hline Polycladida & Callioplana evelinae Marcus 1954 & Ilhabela/SP (Marcus 1954) \\
\hline Polycladida & Candimba divae Marcus 1949 & Guarujá/SP (Marcus 1949) \\
\hline Polycladida & Cestoplana salar Marcus 1949 & Guarujá/SP (Marcus 1949) \\
\hline Polycladida & Cestoplana techa du-Bois-Reymond Marcus 1957 & Ilhabela/SP (Bois-Reymond Marcus 1957) \\
\hline Polycladida & Chromyella saga Corrêa 1958 & Cabo Frio/RJ (Corrêa 1958) \\
\hline Polycladida & Comoplana angusta (Verrill 1893) & Guarujá/SP, São Vicente/SP (Marcus 1947) \\
\hline Polycladida & Cryptocelis lilianae (du Bois-Reymond Marcus 1958) & Ubatuba/SP (du Bois-Reymond Marcus 1958) \\
\hline Polycladida & Cycloporus gabriellae Marcus 1950 & $\begin{array}{l}\text { Ilhabela/SP (Marcus 1950) } \\
\text { Ubatuba/SP (Marcus 1952) }\end{array}$ \\
\hline Polycladida & Distylochus isifer (du Bois Reymond Marcus 1955) & Cananéia/SP (du Bois Reymond Marcus 1955b) \\
\hline Polycladida & Distylochus martae (Marcus 1947) & Guarujá/SP (Marcus 1947) \\
\hline Polycladida & Duplominona tridens (Marcus 1954) & Ilhabela/SP (Marcus 1954) \\
\hline Polycladida & Enchiridium evelinae Marcus 1949 & Guarujá/SP, Ilhabela/SP (Marcus 1949) \\
\hline Polycladida & Euplana hymanae Marcus 1947 & Guarujá/SP (Marcus 1947) \\
\hline Polycladida & Euprosthiostomum mortensenii Marcus 1948 & Santos/SP, São Vicente/SP (Marcus 1948) \\
\hline Polycladida & Eurylepta neptis Du-Bois Reymond Marcus 1955 & Ilhabela/SP (du Bois-Reymond-Marcus 1955b) \\
\hline Polycladida & Eurylepta piscatoria (Marcus 1947) & Guarujá/SP (Marcus 1947) \\
\hline Polycladida & Eurylepta turma Marcus 1952 & Ilhabela/SP, Ubatuba/SP (Marcus 1952) \\
\hline Polycladida & Hoploplana divae Marcus 1950 & Ilhabela/SP (Marcus 1950) \\
\hline Polycladida & Hoploplana usaguia Smith 1960 & Ubatuba/SP (Smith 1960) \\
\hline Polycladida & Interplana evelinae (Marcus 1952) & Guarujá/SP, Itanhaém/SP (Marcus 1952) \\
\hline Polycladida & Itannia ornata Marcus 1947 & $\begin{array}{l}\text { Guarujá/SP (Marcus 1947) } \\
\text { Ilhabela/SP, Ubatuba/SP (Marcus 1952) }\end{array}$ \\
\hline Polycladida & Latocestus brasiliensis Hyman 1955 & São Francisco*/? (Hyman 1955) \\
\hline Polycladida & Lurymare gabriellae (Marcus 1949) & Ilhabela/SP (Marcus 1949) \\
\hline Polycladida & Lurymare matarazzoi (Marcus 1950) & Ilhabela/SP (Marcus 1950) \\
\hline Polycladida & Lurymare utarum (Marcus 1952) & Ilhabela/SP (Marcus 1952) \\
\hline Polycladida & Nonatona euscopa Marcus 1952 & Matinhos/PR (Marcus 1952) \\
\hline Polycladida & Notocomplana evelinae (Marcus 1947) & Guarujá/SP (Marcus 1947) \\
\hline Polycladida & Notocomplana syntoma (Marcus 1947) & São Vicente/SP (Marcus 1947) \\
\hline Polycladida & Notoplana divae Marcus 1948 & Matinhos/PR, Santos/SP (Marcus 1948) \\
\hline Polycladida & Notoplana martae Marcus 1948 & Guarujá/SP, Santos/SP (Marcus 1948) \\
\hline Polycladida & Notoplana megala Marcus 1952 & Ilhabela/SP, Ubatuba/SP (Marcus 1952) \\
\hline Polycladida & Notoplana micheli Marcus 1949 & Itapemirim (Marcus 1949) \\
\hline Polycladida & Notoplana plecta Marcus 1947 & Guarujá/SP (Marcus 1947) \\
\hline Polycladida & Notoplana sawayai Marcus 1947 & Guarujá/SP (Marcus 1947) \\
\hline Polycladida & Notoplana syntoma Marcus 1948 & São Vicente/SP (Marcus 1948) \\
\hline Polycladida & Parviplana lynca (du-Bois Reymond Marcus 1958) & Cananéia/SP (du-Bois Reymond Marcus 1958) \\
\hline Polycladida & Pentaplana divae Marcus 1949 & São Vicente/SP (Marcus 1949) \\
\hline Polycladida & Phaenocelis medvenica Marcus 1952 & Ilhabela/SP (Marcus 1952) \\
\hline Polycladida & Phaenocelis medvenica Marcus 1952 & Ilhabela/SP (Marcus 1952) \\
\hline Polycladida & Prolatocestus ocellatus (Marcus 1947) & Guarujá/SP (Marcus 1947) \\
\hline
\end{tabular}


Tabela 2. Continuação...

\begin{tabular}{|c|c|c|}
\hline Ordem & Espécie & Município/Estado \\
\hline Polycladida & Prosthiostomum cynarium Marcus 1950 & Ilhabela/SP (Marcus 1950) \\
\hline Polycladida & Prosthiostomum gilvum Marcus 1950 & Ilhabela/SP (Marcus 1950) \\
\hline Polycladida & Pseudobiceros evelinae (Marcus 1950) & Santos/SP (Marcus 1950) \\
\hline Polycladida & Pseudoceros chloreus Marcus 1949 & Ilhabela/SP (Marcus 1949) \\
\hline Polycladida & Pseudoceros hispidus du-Bois Reymond Marcus 1955 & Ilhabela/SP (du Bois-Reymond-Marcus 1955b) \\
\hline Polycladida & Pseudoceros mopsus Marcus 1952 & Ilhabela/SP (Marcus 1952) \\
\hline Polycladida & Stylochoplana divae Marcus 1947 & Guarujá/SP (Marcus 1947) \\
\hline Polycladida & Stylochoplana leptalea Marcus 1947 & $\begin{array}{l}\text { Caravelas/BA (Marcus 1968) } \\
\text { Guarujá/SP (Marcus 1947) }\end{array}$ \\
\hline Polycladida & Stylochoplana selenopsis Marcus 1947 & São Vicente/SP (Marcus 1947) \\
\hline Polycladida & $\begin{array}{l}\text { Stylochoplana walsergia du Bois-Reymond Marcus \& } \\
\text { Marcus } 1968\end{array}$ & $\begin{array}{l}\text { Caravelas/BA } \\
\text { (du Bois-Reymond Marcus \& Marcus 1968) }\end{array}$ \\
\hline Polycladida & Stylochus catus du-Bois Reymond Marcus 1958 & $\begin{array}{l}\text { Ubatuba/SP, Ilhabela/SP } \\
\text { (du Bois-Reymond Marcus 1958) }\end{array}$ \\
\hline Polycladida & Stylochus refertus du-Bois Reymond Marcus 1965 & São Vicente/SP (du-Bois Reymond Marcus 1965) \\
\hline Polycladida & Stylochus ticus Marcus 1952 & Ilhabela/SP Ubatuba/SP (Marcus 1952) \\
\hline Polycladida & Theama evelinae Marcus 1949 & Ilhabela/SP (Marcus 1949) \\
\hline Polycladida & Thysanozoon brochii (Risso 1818) & Ilhabela/SP (Marcus 1949) \\
\hline Polycladida & Triadomma curvum Marcus 1949 & Ilhabela/SP (Marcus 1949) \\
\hline Polycladida & Triadomma evelinae Marcus 1947 & Guarujá/SP (Marcus 1947) \\
\hline Polycladida & Zygantroplana henriettae Corrêa 1949 & $\begin{array}{l}\text { Ilhabela/SP Matinhos/PR Itapemirim/ES } \\
\text { (Corrêa 1949) }\end{array}$ \\
\hline Polycladida & Zygantroplana plesia Corrêa 1949 & Antonina/PR (Corrêa 1949) \\
\hline Polycladida & Acerotisa bituna Marcus 1947 & Guarujá/SP (Marcus 1947) \\
\hline Maricola (Tricladida) & Dinizia divae Marcus 1947 & Guarujá/SP (Marcus 1947) \\
\hline Maricola (Tricladida) & Leucolesma corderoi Marcus 1948 & Guarujá/SP, Santos/SP (Marcus 1948) \\
\hline Maricola (Tricladida) & Nerpa evelinae Marcus 1948 & Guarujá/SP, São Vicente/SP (Marcus 1948) \\
\hline Maricola (Tricladida) & Procerodes dahli Marcus \& Marcus 1959 & Haicabe*/? (Sluys 1989) \\
\hline Maricola (Tricladida) & Puiteca camica du Bois-Reymond-Marcus 1955 & Ubatuba/SP (du Bois-Reymond-Marcus 1955b) \\
\hline Maricola (Tricladida) & Tiddles evelinae Marcus 1963 & Cananéia/SP (Marcus 1948) \\
\hline Maricola (Tricladida) & Vatapa gabriellae Marcus 1948 & Guarujá/SP (Marcus 1948) \\
\hline Cavernicola (Tricladida) & Rhodax evelinae Marcus 1946 & São Paulo/SP (Marcus 1946) \\
\hline Paludicola (Tricladida) & Bopsula evelinae Marcus 1946 & Campos do Jordão/SP (Marcus 1946) \\
\hline Paludicola (Tricladida) & Giradia biapertura Sluys 1987 & São Sebastião do Caí/RS (Sluys et al. 1997) \\
\hline Paludicola (Tricladida) & Girardia anderlani (Kawakatsu \& Hauser 1983) & $\begin{array}{l}\text { São Francisco de Paula/RS (Knakievicz et al. 2007) } \\
\text { Caçapava do Sul/RS (Knakievicz et al. 2007) } \\
\text { Dois Irmãos/RS (Kawakatsu et al. 1976) } \\
\text { Encruzilhada do Sul/RS (Kawakatsu et al. 1986) } \\
\text { Erechim/RS (Knakievicz et al. 2007) } \\
\text { Flores da Cunha/RS (Knakievicz et al. 2007) } \\
\text { Salvador do Sul/RS (Knakievicz et al. 2007) } \\
\text { Santa Maria/RS (Knakievicz et al. 2007) } \\
\text { São Leopoldo/RS (Kawakatsu et al. 1983a) } \\
\text { Severiano de Almeida/RS (Knakievicz et al. 2007) } \\
\text { Uruguaiana/RS (Knakievicz et al. 2007) } \\
\text { Viamão/RS (Knakievicz et al. 2007) } \\
\text { Pelotas/RS (Kawakatsu et al. 1986) }\end{array}$ \\
\hline Paludicola (Tricladida) & Girardia arndti Marcus 1946 & $\begin{array}{l}\text { Campos de Jordão/RJ (Marcus 1946) } \\
\text { Morro Reuter/RS (Kawakatsu et al. 1986) }\end{array}$ \\
\hline
\end{tabular}


Tabela 2. Continuação...

\begin{tabular}{|c|c|c|}
\hline Ordem & Espécie & Município/Estado \\
\hline Paludicola (Tricladida) & Girardia chilla (Marcus 1954) & Salesópolis/SP (Sluys 1996) \\
\hline Paludicola (Tricladida) & Girardia festae (Borelli 1898) & Urucum*/MT (Borelli 1898) \\
\hline Paludicola (Tricladida) & Girardia hypoglauca (Marcus 1948) & São Paulo/SP (Marcus 1948) \\
\hline Paludicola (Tricladida) & Girardia nonatoi (Marcus 1946) & São Paulo/SP (Marcus 1946) \\
\hline Paludicola (Tricladida) & Girardia paramensis (Fuhrman 1914) & $\begin{array}{l}\text { Medicilândia/PA, Altamira/PA } \\
\text { (Kawakatsu \& E.M. Froehlich 1992) }\end{array}$ \\
\hline Paludicola (Tricladida) & Girardia schubarti (Marcus 1946) & $\begin{array}{l}\text { Botucatu/SP (Kawakatsu et al. 1983) } \\
\text { Campos do Jordão/SP (Marcus 1946) } \\
\text { Monte Alegre/SP (Marcus 1946) } \\
\text { São Carlos/SP (Kawakatsu et al. 1982) } \\
\text { Salesópolis/SP (Kawakatsu et al. 1976) } \\
\text { São Paulo/SP (Marcus 1946) } \\
\text { Nova Petrópolis/RS (Kawakatsu et al. 1980) } \\
\text { Caxias do Sul/RS (Kawakatsu et al. 1980) } \\
\text { Dois Irmãos/RS (Hensel 1980) } \\
\text { Morro Reuter/RS (Kawakatsu et al. 1980) } \\
\text { Erechim/RS (Knakievicz et al. 2007) } \\
\text { Flores da Cunha/RS (Knakievicz et al. 2007) } \\
\text { Harmonia/RS (Kawakatsu et al. 1980) } \\
\text { Jacutinga/RS (Knakievicz et al. 2007) } \\
\text { Júlio de Castilhos/RS (Kawakatsu et al. 1980) } \\
\text { Montenegro/RS (Hensel 1980) } \\
\text { Morro Reuter/RS (Kawakatsu et al. 1980) } \\
\text { Novo Hamburgo/RS (Kawakatsu et al. 1980) } \\
\text { Picada Café/RS (Kawakatsu et al. 1980) } \\
\text { Santa Maria/RS (Knakievicz et al. 2007) } \\
\text { Santo Ângelo/RS (Knakievicz et al. 2007) } \\
\text { São Francisco de Paula/RS (Kawakatsu et al. 1986) } \\
\text { São José dos Ausentes/RS (Knakievicz et al. 2007) } \\
\text { São Leopoldo/RS (Kawakatsu et al. 1976) } \\
\text { Sapiranga/RS (Hensel 1980) } \\
\text { Severiano de Almeida/RS (Knakievicz et al. 2007) } \\
\text { Uruguaiana/RS (Knakievicz et al. 2007) } \\
\text { Pelotas/RS (Kawakatsu et al. 1986) }\end{array}$ \\
\hline Paludicola (Tricladida) & Girardia tigrina (Girard 1850) & $\begin{array}{l}\text { Salvador/BA (Preza \& Smith 2001) } \\
\text { São Carlos/SP (Kawakatsu et al. 1982) } \\
\text { Botucatu/SP (Kawakatsu et al. 1983b) } \\
\text { São Paulo/SP (Marcus 1946) } \\
\text { Arambaré/RS (Knakievicz et al. 2007) } \\
\text { Arroio do Meio/RS (Kawakatsu et al. 1992) } \\
\text { Salvador do Sul/RS (Kawakatsu et al. 1992) } \\
\text { Glorinha/RS (Knakievicz et al. 2007) } \\
\text { Porto Alegre/RS (Marcus 1946) } \\
\text { São Francisco de Paula/RS (Kawakatsu et al. 1986) } \\
\text { São Leopoldo/RS (Kawakatsu et al. 1980) } \\
\text { Pelotas/RS (Kawakatsu et al. 1986) }\end{array}$ \\
\hline Paludicola (Tricladida) & $\begin{array}{l}\text { Girardia ururiograndeana (Kawakatsu, Hause \& Ponce } \\
\text { de Leon 1992) }\end{array}$ & $\begin{array}{l}\text { Arroio do Meio/RS Arroio Grande/RS } \\
\text { (Kawakatsu et al. 1992) }\end{array}$ \\
\hline Terricola (Tricladida) & Amaga righii (E.M. Froehlich 1972) & $\begin{array}{l}\text { Serra do Navio/AP } \\
\text { (E.M. Froehlich \& Froehlich 1972) }\end{array}$ \\
\hline Terricola (Tricladida) & Bipalium kewense Moseley 1878 & exótica e cosmopolita \\
\hline Terricola (Tricladida) & $\begin{array}{l}\text { Cephaloflexa araucariana Carbayo \& Leal-Zanchet } \\
2003\end{array}$ & $\begin{array}{l}\text { São Francisco de Paula/RS } \\
\text { (Carbayo \& Leal-Zanchet 2003) }\end{array}$ \\
\hline
\end{tabular}


Tabela 2. Continuação...

\begin{tabular}{|c|c|c|}
\hline Ordem & Espécie & Município/Estado \\
\hline Terricola (Tricladida) & Cephaloflexa bergi (Graff 1899) & $\begin{array}{l}\text { Teresópolis/RJ (Schirch 1929) } \\
\text { Ubatuba/SP (Froehlich 1956a) } \\
\text { São Paulo/SP (Graff 1899) } \\
\text { Santo André/SP (Marcus 1951) } \\
\text { Mongaguá/SP (Marcus 1951) } \\
\text { Santos/SP (Marcus 1951) } \\
\text { Curitiba/PR (Froehlich 1956b) } \\
\text { Paranaguá/PR (Froehlich 1956b) } \\
\text { Morretes/PR (Froehlich 1956b) } \\
\text { Blumenau/SC (Froehlich 1956b) } \\
\text { Cambará do Sul/RS (Fick et al. 2006) }\end{array}$ \\
\hline Terricola (Tricladida) & Choeradoplana bilix Marcus 1951 & Angra dos Reis/RJ (Marcus 1951) \\
\hline Terricola (Tricladida) & Choeradoplana catua Froehlich 1955 & Teresópolis/RJ (Froehlich 1955a) \\
\hline Terricola (Tricladida) & Choeradoplana ehrenreichi Graff 1899 & Joinville/SC (Graff 1899) \\
\hline Terricola (Tricladida) & Choeradoplana iheringi Graff 1899 & $\begin{array}{l}\text { Nova Lima/MG (Riester 1938) } \\
\text { Teresópolis/RJ (Graff 1899) } \\
\text { Salesópolis/SP (Souza \& Leal-Zanchet 2003) } \\
\text { Ribeirão Pires/SP (Riester 1938) } \\
\text { Cambará do Sul/RS (Fick et al. 2006) } \\
\text { São Paulo/SP (Riester 1938) } \\
\text { São Francisco de Paula/RS } \\
\text { (Souza \& Leal-Zanchet 2003) } \\
\text { Taquara/RS (Graff 1899)São Leopoldo/RS } \\
\text { (Froehlich 1959) }\end{array}$ \\
\hline Terricola (Tricladida) & Choeradoplana langi (Graff 1894) & $\begin{array}{l}\text { Blumenau/SC (Graff 1899) } \\
\text { Brusque/SC (Marcus 1951) }\end{array}$ \\
\hline Terricola (Tricladida) & Choeradoplana marthae Froehlich 1955 & Mongaguá/ SP (Froehlich 1955a) \\
\hline Terricola (Tricladida) & Diporodemus hymanae Froehlich 1972 & Serra do Navio/AP (Froehlich \& Froehlich 1972) \\
\hline Terricola (Tricladida) & Dolichoplana carvalhoi Corrêa 1947 & exótica e cosmopolita \\
\hline Terricola (Tricladida) & Endeavouria septemlineata (Hyman 1939) & $\begin{array}{l}\text { exótica } \\
\text { São Paulo/SP, Campo Grande/MS, } \\
\text { Florianópolis/SC, Maquiné/RS, } \\
\text { São Lourenço do Sul/RS (Carbayo et al. 2008) }\end{array}$ \\
\hline Terricola (Tricladida) & Enterosyringa pseudorhynchodemus (Riester 1938) & $\begin{array}{l}\text { Teresópolis/RJ (Riester 1938) } \\
\text { São Paulo/SP (Marcus 1951) } \\
\text { Blumenau/SC (Froehlich 1959) }\end{array}$ \\
\hline Terricola (Tricladida) & Geobia subterranea Schultze \& Müller 1857 & $\begin{array}{l}\text { Teresópolis/RJ (Riester 1938) } \\
\text { Mogi das Cruzes/SP (Marcus 1951) } \\
\text { São Paulo/SP (Marcus 1951) } \\
\text { Avaré/SP (Marcus 1951) } \\
\text { Blumenau/SC (Schultze \& Müller 1857) }\end{array}$ \\
\hline Terricola (Tricladida) & Geoplana (Barreirana) barreirana Riester 1938 & $\begin{array}{l}\text { Teresópolis/RJ (Riester 1938) } \\
\text { Rio de Janeiro/RJ (Froehlich 1956a) } \\
\text { Ubatuba/SP (Froehlich 1956a) }\end{array}$ \\
\hline Terricola (Tricladida) & Geoplana (Barreirana) cafusa Froehlich 1956 & Ubatuba/SP (Froehlich 1956a) \\
\hline Terricola (Tricladida) & Geoplana (Barreirana) cassula E.M. Froehlich 1955 & Teresópolis/RJ (E.M. Froehlich 1955a) \\
\hline Terricola (Tricladida) & Geoplana (Barreirana) elegans (Darwin 1844) & Rio de Janeiro/RJ (Darwin 1844) \\
\hline Terricola (Tricladida) & Geoplana (Barreirana) zebroides Riester 1938 & Teresópolis/RJ (Riester 1938) \\
\hline Terricola (Tricladida) & Geoplana (Geoplana) apeva Froehlich 1959 & Brusque/SC, Blumenau/SC (Froehlich 1959) \\
\hline Terricola (Tricladida) & Geoplana (Geoplana) applanata Graff 1899 & $\begin{array}{l}\text { Nova Lima/MG (Riester 1938) } \\
\text { Santo Antônio de Pádua/RJ (Graff 1899) } \\
\text { Teresópolis/RJ (Riester 1938) }\end{array}$ \\
\hline Terricola (Tricladida) & Geoplana (Geoplana) argus Graff 1899 & Nova Friburgo/RJ (Graff 1899) \\
\hline Terricola (Tricladida) & Geoplana (Geoplana) arpi Schirch 1929 & Maylasky/RJ (Schirch 1929) \\
\hline
\end{tabular}


Tabela 2. Continuação...

\begin{tabular}{|c|c|c|}
\hline Ordem & Espécie & Município/Estado \\
\hline Terricola (Tricladida) & Geoplana (Geoplana) assu Froehlich 1959 & Blumenau/SC, Pomerode/SC (Froehlich 1959) \\
\hline Terricola (Tricladida) & Geoplana (Geoplana) beckeri Froehlich 1959 & Salvador/BA (Froehlich 1959) \\
\hline Terricola (Tricladida) & Geoplana (Geoplana) blaseri Schirch 1929 & RJ*, ES* (Schirch 1929) \\
\hline Terricola (Tricladida) & Geoplana (Geoplana) braunsi Graff 1899 & $\begin{array}{l}\text { Amparo/SP (Froehlich 1958) } \\
\text { Santos/SP (Graff 1899) } \\
\text { Mogi das Cruzes/SP (Marcus 1951) }\end{array}$ \\
\hline Terricola (Tricladida) & Geoplana (Geoplana) bresslaui Schirch 1929 & Teresópolis/RJ (Schirch 1929) \\
\hline Terricola (Tricladida) & $\begin{array}{l}\text { Geoplana (Geoplana) burmeisteri Schultze \& Müller } \\
1857\end{array}$ & $\begin{array}{l}\text { Teresópolis/RJ (Schirch 1929) } \\
\text { Rio de Janeiro/RJ (Schultze \& Müller 1857) } \\
\text { Ubatuba/SP (Froehlich 1956a) } \\
\text { São Paulo/SP (Marcus 1951) } \\
\text { Matinhos/PR (Froehlich 1956b) } \\
\text { Blumenau/SC (Froehlich 1956b) } \\
\text { Itajaí/SC (Froehlich 1956b) } \\
\text { Brusque/SC (Froehlich 1956b) }\end{array}$ \\
\hline Terricola (Tricladida) & Geoplana (Geoplana) caapora Froehlich 1958 & Apiaí/SP (Froehlich 1958) \\
\hline Terricola (Tricladida) & Geoplana (Geoplana) carinata Riester 1938 & $\begin{array}{l}\text { Ribeirão Pires/SP (Riester 1938) } \\
\text { Juquiá/SP (Froehlich 1958) } \\
\text { São Paulo/SP (Marcus 1951) } \\
\text { Santos/SP (Marcus 1951) } \\
\text { Mongaguá/SP (Marcus 1951) } \\
\text { Pirassununga/SP (Froehlich 1958) } \\
\text { Mogi das Cruzes/SP (Marcus 1951) } \\
\text { Curitiba/PR (Froehlich 1956b) } \\
\text { Morretes/PR (Froehlich 1956b) }\end{array}$ \\
\hline Terricola (Tricladida) & Geoplana (Geoplana) carrierei Graff 1897 & $\begin{array}{l}\text { São Paulo/SP (Marcus 1951) } \\
\text { Santo André/SP (Marcus 1951) } \\
\text { São Leopoldo/RS (Froehlich 1959) }\end{array}$ \\
\hline Terricola (Tricladida) & Geoplana (Geoplana) catharina Hyman 1957 & São Bento do Sul/SC (Hyman 1955) \\
\hline Terricola (Tricladida) & Geoplana (Geoplana) chita Froehlich 1956 & $\begin{array}{l}\text { Eldorado/SP (Froehlich 1956b) } \\
\text { Curitiba/PR (Froehlich 1956b) }\end{array}$ \\
\hline Terricola (Tricladida) & Geoplana (Geoplana) chiuna E.M. Froehlich 1955 & Pirassununga/SP (E.M. Froehlich 1955a) \\
\hline Terricola (Tricladida) & Geoplana (Geoplana) crioula E.M. Froehlich 1955 & São Paulo/SP (E.M. Froehlich 1955a) \\
\hline Terricola (Tricladida) & Geoplana (Geoplana) dictyonota Riester 1938 & Araras*/MG (Riester 1938) \\
\hline Terricola (Tricladida) & Geoplana (Geoplana) divae Marcus 1951 & $\begin{array}{l}\text { Alto da Serra/SP (Marcus 1951) } \\
\text { Vila Atlântica/SP (Marcus 1951) }\end{array}$ \\
\hline Terricola (Tricladida) & $\begin{array}{l}\text { Geoplana (Geoplana) eudoxiae Ogren \& Kawakatsu } \\
1990\end{array}$ & Teresópolis/RJ (Riester 1938) \\
\hline Terricola (Tricladida) & $\begin{array}{l}\text { Geoplana (Geoplana) eudoximariae Ogren \& Kawakatsu } \\
1990\end{array}$ & Teresópolis/RJ (Riester 1938) \\
\hline Terricola (Tricladida) & Geoplana (Geoplana) evelinae Marcus 1951 & $\begin{array}{l}\text { Mongaguá/SP (Marcus 1951) } \\
\text { Santo André/SP (Marcus 1951) }\end{array}$ \\
\hline Terricola (Tricladida) & Geoplana (Geoplana) ferussaci Graff 1899 & $\begin{array}{l}\text { Nova Lima/MG (Riester 1938) } \\
\text { Araras/MG (Riester 1938) } \\
\text { Perto de Rio de Janeiro*/RJ } \\
\text { Teresópolis/RJ (Schirch 1929) } \\
\text { Ubatuba/SP (Froehlich 1956a) } \\
\text { São Paulo/SP (Marcus 1951) } \\
\text { Mogi das Cruzes/SP (Marcus 1951) } \\
\text { Blumenau/SC (Graff 1899) } \\
\text { RS* (Graff 1899) }\end{array}$ \\
\hline Terricola (Tricladida) & Geoplana (Geoplana) fragai Froehlich 1955 & Teresópolis/RJ (Froehlich 1955b) \\
\hline Terricola (Tricladida) & Geoplana (Geoplana) fryi Graff 1899 & Teresópolis/RJ (Schirch 1929) \\
\hline
\end{tabular}


Tabela 2. Continuação...

\begin{tabular}{|c|c|c|}
\hline Ordem & Espécie & Município/Estado \\
\hline Terricola (Tricladida) & Geoplana (Geoplana) gaucha Froehlich 1959 & $\begin{array}{l}\text { Rio de Janeiro/RJ (Graff 1899) } \\
\text { Salvador do Sul/RS (Froehlich 1959) } \\
\text { São Leopoldo/RS (Antunes et al. 2008) } \\
\text { Porto Alegre/RS (Froehlich 1959) } \\
\text { Viamão/RS (Antunes et al. 2008) }\end{array}$ \\
\hline Terricola (Tricladida) & Geoplana (Geoplana) glieschi Froehlich 1959 & Iraí/RS (Froehlich 1959) \\
\hline Terricola (Tricladida) & Geoplana (Geoplana) goettei Schirch 1929 & Teresópolis/RJ (E.M. Froehlich 1955a) \\
\hline Terricola (Tricladida) & Geoplana (Geoplana) hina Marcus 1951 & Santo André/SP (Marcus 1951) \\
\hline Terricola (Tricladida) & Geoplana (Geoplana) incognita Riester 1938 & Teresópolis/RJ (Riester 1938) \\
\hline Terricola (Tricladida) & Geoplana (Geoplana) itatiayana Schirch 1929 & $\begin{array}{l}\text { Resende/RJ (Schirch 1929) } \\
\text { São Paulo/SP (Marcus 1951) }\end{array}$ \\
\hline Terricola (Tricladida) & Geoplana (Geoplana) jandira Froehlich 1955 & Teresópolis/RJ (Froehlich 1955b) \\
\hline Terricola (Tricladida) & Geoplana (Geoplana) joia Froehlich 1956 & São José dos Pinhais/PR (Froehlich 1956b) \\
\hline Terricola (Tricladida) & Geoplana (Geoplana) ladislavii Graff 1899 & $\begin{array}{l}\text { Blumenau/SC (Froehlich 1959) } \\
\text { Cambará do Sul/RS (Fick et al. 2006) } \\
\text { São Francisco de Paula/RS (Carbayo et al. 2002) } \\
\text { São Leopoldo/RS (Froehlich 1959) } \\
\text { Taquara/RS (Graff 1899) } \\
\text { Santa Maria/RS (Castro et al. 2005) } \\
\text { Viamão/RS (Antunes et al. 2008) }\end{array}$ \\
\hline Terricola (Tricladida) & Geoplana (Geoplana) livia E.M. Froehlich 1955 & São Paulo/SP (E.M. Froehlich 1955a) \\
\hline Terricola (Tricladida) & $\begin{array}{l}\text { Geoplana (Geoplana) marginata Schultze \& Müller } \\
1857\end{array}$ & Blumenau/SC (Schultze \& Müller 1857) \\
\hline Terricola (Tricladida) & $\begin{array}{l}\text { Geoplana (Geoplana) marmorata Schultze \& Müller } \\
1857\end{array}$ & $\begin{array}{l}\text { Blumenau/SC (Schultze \& Müller 1857) } \\
\text { Pomerode/SC (Froehlich 1959) }\end{array}$ \\
\hline Terricola (Tricladida) & Geoplana (Geoplana) matuta E.M. Froehlich 1955 & Teresópolis/RJ (E.M. Froehlich 1955a) \\
\hline Terricola (Tricladida) & Geoplana (Geoplana) metzi Graff 1899 & $\begin{array}{l}\text { Rio de Janeiro/RJ (Riester 1938) } \\
\text { São Paulo/SP (Graff 1899) } \\
\text { Ribeirão Pires/SP (Marcus 1951) }\end{array}$ \\
\hline Terricola (Tricladida) & Geoplana (Geoplana) mirim E.M. Froehlich 1972 & $\begin{array}{l}\text { Serra do Navio/AP } \\
\text { (Froehlich \& E.M. Froehlich 1972) }\end{array}$ \\
\hline Terricola (Tricladida) & Geoplana (Geoplana) multicolor Graff 1899 & $\begin{array}{l}\text { Teresópolis/RJ (Froehlich 1956a) } \\
\text { Santo André/SP (Marcus 1951) } \\
\text { Avaré/SP (Marcus 1951) } \\
\text { Mogi das Cruzes/SP (Marcus 1951) } \\
\text { São Paulo/SP (Graff 1899) } \\
\text { Curitiba/PR (Froehlich 1956b) } \\
\text { Guapiara/PR (Froehlich 1958) } \\
\text { Ponta Grossa/PR (Froehlich 1958) } \\
\text { Lapa/PR (Froehlich 1956b) }\end{array}$ \\
\hline Terricola (Tricladida) & Geoplana (Geoplana) nigra Froehlich 1959 & Taquara/RS (Froehlich 1959) \\
\hline Terricola (Tricladida) & Geoplana (Geoplana) pavani Marcus 1951 & $\begin{array}{l}\text { Santo André/SP (Froehlich 1955c) } \\
\text { Mogi das Cruzes/SP (Marcus 1951) }\end{array}$ \\
\hline Terricola (Tricladida) & Geoplana (Geoplana) phocaica Marcus 1951 & São Paulo/SP (Marcus 1951) \\
\hline Terricola (Tricladida) & Geoplana (Geoplana) picta Froehlich 1956 & Ubatuba/SP (Froehlich 1956a) \\
\hline Terricola (Tricladida) & Geoplana (Geoplana) poca Froehlich 1958 & Itanhaém/SP (Froehlich 1958) \\
\hline Terricola (Tricladida) & Geoplana (Geoplana) polyophthalma Graff 1899 & $\begin{array}{l}\text { Matinhos/PR (Froehlich 1956b) } \\
\text { Blumenau/SC (Graff 1899) } \\
\text { Joinville/SC (Graff 1899) } \\
\text { Pomerode/SC (Froehlich 1956b) } \\
\text { Taquara/RS (Graff 1899) }\end{array}$ \\
\hline Terricola (Tricladida) & Geoplana (Geoplana) preta Riester 1938 & $\begin{array}{l}\text { Teresópolis/RJ (Riester 1938) } \\
\text { São Paulo/SP (Marcus 1951) }\end{array}$ \\
\hline
\end{tabular}

São Paulo/SP (Marcus 1951) 
Tabela 2. Continuação...

\begin{tabular}{|c|c|c|}
\hline Ordem & Espécie & Município/Estado \\
\hline Terricola (Tricladida) & $\begin{array}{l}\text { Geoplana (Geoplana) pseudovaginuloides Riester } \\
1938\end{array}$ & Teresópolis/RJ (Riester 1938) \\
\hline Terricola (Tricladida) & Geoplana (Geoplana) quagga Marcus 1951 & $\begin{array}{l}\text { Teresópolis/RJ (Froehlich 1956a) } \\
\text { São Paulo/SP (Marcus 1951) } \\
\text { Blumenau/SC (Froehlich 1959) }\end{array}$ \\
\hline Terricola (Tricladida) & Geoplana (Geoplana) regia E.M. Froehlich 1955 & Araraquara/SP (E.M. Froehlich 1955a) \\
\hline Terricola (Tricladida) & Geoplana (Geoplana) riesteri Froehlich 1955 & $\begin{array}{l}\text { Araras/MG (Riester 1938) } \\
\text { Teresópolis/RJ (Riester 1938) }\end{array}$ \\
\hline Terricola (Tricladida) & $\begin{array}{l}\text { Geoplana (Geoplana) rufiventris Schultze \& Müller } \\
1857\end{array}$ & Blumenau/SC (Schultze \& Müller 1857) \\
\hline Terricola (Tricladida) & Geoplana (Geoplana) ruiva E.M. Froehlich 1972 & $\begin{array}{l}\text { Serra do Navio/AP } \\
\text { (Froehlich \& E.M. Froehlich 1972) }\end{array}$ \\
\hline Terricola (Tricladida) & Geoplana (Geoplana) schubarti Froehlich 1958 & Itanhaém/SP, São Paulo/SP (Froehlich 1958) \\
\hline Terricola (Tricladida) & Geoplana (Geoplana) suva Froehlich 1959 & Blumenau/SC (Froehlich 1959) \\
\hline Terricola (Tricladida) & Geoplana (Geoplana) tamoia E.M. Froehlich 1955 & Teresópolis/RJ (E.M. Froehlich 1955a) \\
\hline Terricola (Tricladida) & Geoplana (Geoplana) tapira Froehlich 1958 & Tapiraí/SP (Froehlich 1958) \\
\hline Terricola (Tricladida) & Geoplana (Geoplana) taxiarcha Marcus 1951 & $\begin{array}{l}\text { São Paulo/SP (Marcus 1951) } \\
\text { Mogi das Cruzes/SP (Marcus 1951) } \\
\text { Ubatuba/SP (Froehlich, 1956a) }\end{array}$ \\
\hline Terricola (Tricladida) & Geoplana (Geoplana) toriba Froehlich 1958 & Juquiá/SP (Froehlich 1958) \\
\hline Terricola (Tricladida) & Geoplana (Geoplana) trigueira E.M. Froehlich 1955 & $\begin{array}{l}\text { Teresópolis/RJ (E.M. Froehlich 1955a) } \\
\text { Ubatuba/SP (Froehlich 1956a) }\end{array}$ \\
\hline Terricola (Tricladida) & Geoplana (Geoplana) tихаиа E.M. Froehlich 1955 & São Paulo/SP (E.M. Froehlich 1955a) \\
\hline Terricola (Tricladida) & Geoplana (Geoplana) vaginuloides (Darwin 1844) & $\begin{array}{l}\text { Teresópolis/RJ (Riester 1938) } \\
\text { Rio de Janeiro/RJ (Darwin 1844) } \\
\text { São Paulo/SP (Marcus 1952) } \\
\text { Itanhaém/SP (Froehlich 1958) } \\
\text { Eldorado/SP (Marcus 1951) }\end{array}$ \\
\hline Terricola (Tricladida) & Geoplana (Geoplana) yara E.M. Froehlich 1955 & Teresópolis/RJ (E.M. Froehlich 1955a) \\
\hline Terricola (Tricladida) & Geoplana franciscana Leal-Zanchet \& Carbayo 2000 & $\begin{array}{l}\text { São Francisco de Paula/RS } \\
\text { (Zanchet \& Carbayo 2000) } \\
\text { Cambará do Sul/RS (Fick et al. 2006) }\end{array}$ \\
\hline Terricola (Tricladida) & Geoplana josefi Carbayo \& Leal-Zanchet 2001 & $\begin{array}{l}\text { São Francisco de Paula/RS } \\
\text { (Carbayo \& Leal-Zanchet 2001) } \\
\text { Cambará do Sul/RS (Fick et al. 2006) }\end{array}$ \\
\hline Terricola (Tricladida) & $\begin{array}{l}\text { Geoplana rubidolineata Baptista \& Leal-Zanchet } \\
2005\end{array}$ & $\begin{array}{l}\text { São Francisco de Paula/RS } \\
\text { (Baptista \& Leal-Zanchet 2005) }\end{array}$ \\
\hline Terricola (Tricladida) & Issoca jandaia Froehlich 1955 & São Paulo/SP (Froehlich 1955a) \\
\hline Terricola (Tricladida) & Issoca piranga Froehlich 1955 & Teresópolis/RJ (Froehlich 1955a) \\
\hline Terricola (Tricladida) & Issoca potyra Froehlich 1956 & Eldorado/SP (Froehlich 1958) \\
\hline Terricola (Tricladida) & Issoca rezendei (Schirch 1929) & $\begin{array}{l}\text { Teresópolis/RJ (Schirch 1929) } \\
\text { São Paulo/SP (Marcus 1951) } \\
\text { Ubatuba/SP (Froehlich 1955a) } \\
\text { Mongaguá/SP (Froehlich 1955a) } \\
\text { Itanhaém/SP (Froehlich 1955a) } \\
\text { Paranaguá/SP (Froehlich 1955a) } \\
\text { Itajaí/SC (Froehlich 1955a) } \\
\text { Blumenau/SC (Froehlich 1955a) }\end{array}$ \\
\hline Terricola (Tricladida) & Issoca spatulata (Graff 1899) & Blumenau/SC (Graff 1899) \\
\hline
\end{tabular}


Tabela 2. Continuação...

\begin{tabular}{|c|c|c|}
\hline Ordem & Espécie & Município/Estado \\
\hline Terricola (Tricladida) & Kontikia orana Froehlich 1955 & $\begin{array}{l}\text { exótica } \\
\text { Serra do Navio/AP } \\
\text { (Froehlich \& E.M. Froehlich 1972) } \\
\text { Piraí/RJ (Froehlich 1955a) } \\
\text { Ubatuba/SP (du Bois-Reymond-Marcus 1955b) } \\
\text { São Paulo/SP (Froehlich 1955a) } \\
\text { Itanhaém/SP (Froehlich 1955a) } \\
\text { Mongaguá/ SP (Froehlich 1955a) } \\
\text { Pirassununga/SP (Froehlich 1955a) } \\
\text { Baía de Paranaguá/PR (Froehlich 1955a) } \\
\text { Blumenau (Froehlich 1955a) }\end{array}$ \\
\hline Terricola (Tricladida) & Notogynaphallia abundans (Graff 1899) & $\begin{array}{l}\text { São Leopoldo/RS (Antunes et al. 2008) } \\
\text { Taquara/RS (Leal-Zanchet \& E.M. Froehlich 2006) } \\
\text { Poço das Antas/RS } \\
\text { (Leal-Zanchet \& E.M. Froehlich 2006) } \\
\text { Novo Hamburgo/RS } \\
\text { (Leal-Zanchet \& E.M. Froehlich 2006) } \\
\text { Tupandi/RS (Leal-Zanchet \& E.M. Froehlich 2006) } \\
\text { Campo Bom/RS } \\
\text { (Leal-Zanchet \& E.M. Froehlich 2006) } \\
\text { Salvador do Sul/RS } \\
\text { (Leal-Zanchet \& E.M. Froehlich 2006) } \\
\text { Glorinha/RS (Leal-Zanchet \& E.M. Froehlich 2006) } \\
\text { Viamão/RS (Antunes et al. 2008) }\end{array}$ \\
\hline Terricola (Tricladida) & Notogynaphallia albonigra (Riester 1938) & Teresópolis/RJ (Riester 1938) \\
\hline Terricola (Tricladida) & Notogynaphallia atra (Schultze \& Müller 1857) & $\begin{array}{l}\text { Blumenau/SC (Schultze \& Müller 1857) } \\
\text { Pomerode/SC (Froehlich 1956b) } \\
\text { Brusque/SC (Froehlich 1956b) } \\
\text { Lapa/PR (Froehlich 1956b) }\end{array}$ \\
\hline Terricola (Tricladida) & Notogynaphallia caissara (E.M. Froehlich 1955) & $\begin{array}{l}\text { Teresópolis/RJ (E.M. Froehlich 1955) } \\
\text { Rio de Janeiro/RJ (Carbayo 2006) } \\
\text { Ubatuba/SP (E.M. Froehlich 1955) } \\
\text { Ribeirão Pires/SP (Carbayo 2006) } \\
\text { Itanhaém/SP (Carbayo 2006) }\end{array}$ \\
\hline Terricola (Tricladida) & $\begin{array}{l}\text { Notogynaphallia ceciliae E.M. Froehlich \& Leal-Zanchet } \\
2003\end{array}$ & $\begin{array}{l}\text { São Francisco de Paula/RS } \\
\text { (Froehlich \& Leal-Zanchet 2003) }\end{array}$ \\
\hline Terricola (Tricladida) & $\begin{array}{l}\text { Notogynaphallia ernesti Leal-Zanchet \& E.M. Froelhich } \\
2006\end{array}$ & $\begin{array}{l}\text { Jundiaí/SP (Leal-Zanchet \& E.M. Froehlich 2006) } \\
\text { São Paulo/SP (Marcus 1951) } \\
\text { Curitiba (Leal-Zanchet \& E.M. Froehlich 2006) } \\
\text { São Francisco de Paula/RS } \\
\text { (Leal-Zanchet \& E.M. Froehlich 2006) }\end{array}$ \\
\hline Terricola (Tricladida) & Notogynaphallia fita (Froehlich 1959) & Blumenau/SC (Froehlich 1959) \\
\hline Terricola (Tricladida) & $\begin{array}{l}\text { Notogynaphallia froehlichae Ogren \& Kawakatsu } \\
1990\end{array}$ & João Pessoa/PB (Riester 1938) \\
\hline Terricola (Tricladida) & Notogynaphallia goetschi (Riester 1938) & $\begin{array}{l}\text { Teresópolis/RJ (Riester 1938) } \\
\text { Ubatuba/SP (Froehlich 1956a) } \\
\text { São Paulo/SP (Marcus 1951) } \\
\text { Santo André/SP (Marcus 1951) } \\
\text { Mogi das Cruzes/SP (Marcus 1951) } \\
\text { Mongaguá/SP (Marcus 1951) } \\
\text { Guapiara/SP (Froehlich 1958) } \\
\text { Eldorado/SP (Marcus 1951) }\end{array}$ \\
\hline
\end{tabular}


Tabela 2. Continuação...

\begin{tabular}{|c|c|c|}
\hline Ordem & Espécie & Município/Estado \\
\hline Terricola (Tricladida) & $\begin{array}{l}\text { Notogynaphallia graffi Leal-Zanchet \& } \\
\text { E.M. Froehlich } 2006\end{array}$ & $\begin{array}{l}\text { Cambará do Sul/RS (Fick et al. 2006) } \\
\text { São Francisco de Paula/RS } \\
\text { (Leal-Zanchet \& E.M. Froehlich 2006) } \\
\text { Salvador do Sul/RS } \\
\text { (Leal-Zanchet \& E.M. Froehlich 2006) } \\
\text { Três Coroas (Leal-Zanchet \& E.M. Froehlich 2006) }\end{array}$ \\
\hline Terricola (Tricladida) & $\begin{array}{l}\text { Notogynaphallia guaiana Leal-Zanchet \& } \\
\text { Carbayo } 2000\end{array}$ & $\begin{array}{l}\text { São Francisco de Paula/RS } \\
\text { (Zanchet \& Carbayo 2000) }\end{array}$ \\
\hline Terricola (Tricladida) & Notogynaphallia mourei (Froehlich 1956) & $\begin{array}{l}\text { Curitiba/PR (Froehlich 1956b) } \\
\text { Serra do Mar*, entre Curitiba e Paranaguá/PR } \\
\text { (Froehlich 1956b) }\end{array}$ \\
\hline Terricola (Tricladida) & Notogynaphallia muelleri (Diesing 1861) & $\begin{array}{l}\text { Santo André/SP (Froehlich 1959) } \\
\text { Blumenau/SC (Schultze \& Müller 1857) } \\
\text { Pomerode/SC (Froehlich 1959) } \\
\text { Itajaí/SC (Froehlich 1959) } \\
\text { Brusque/SC (Froehlich 1959) }\end{array}$ \\
\hline Terricola (Tricladida) & Notogynaphallia nataliae (Froehlich 1959) & Blumenau/SC (Froehlich 1959) \\
\hline Terricola (Tricladida) & Notogynaphallia octostriata (Schultze \& Müller 1857) & $\begin{array}{l}\text { Teresópolis/RJ (Schirch 1929) } \\
\text { Blumenau/SC (Schultze \& Müller 1857) }\end{array}$ \\
\hline Terricola (Tricladida) & Notogynaphallia parca (E.M. Froehlich 1955) & São Paulo/SP (E.M. Froehlich 1955a) \\
\hline Terricola (Tricladida) & Notogynaphallia plumbea (Froehlich 1956) & $\begin{array}{l}\text { São Paulo/SP (Froehlich 1956b) } \\
\text { Serra do Mar*, entre Curitiba/PR } \\
\text { e Paranaguá/PR (Froehlich 1956b) }\end{array}$ \\
\hline Terricola (Tricladida) & Notogynaphallia sexstriata (Graff 1899) & $\begin{array}{l}\text { Teresópolis/RJ (Schirch 1929) } \\
\text { São Paulo/SP (du Bois-Reymond Marcus 1951b) } \\
\text { Brusque/SC (du Bois-Reymond Marcus 1951b) } \\
\text { Taquara/RS (Graff 1899) }\end{array}$ \\
\hline Terricola (Tricladida) & Pasipha astraea (Marcus 1951) & $\begin{array}{l}\text { Mogi das Cruzes/SP (Marcus 1951) } \\
\text { São Paulo/SP (Marcus 1951) }\end{array}$ \\
\hline Terricola (Tricladida) & Pasipha biseminalis (Riester 1938) & Teresópolis/RJ (Riester 1938) \\
\hline Terricola (Tricladida) & Pasipha caeruleonigra (Riester 1938) & Teresópolis/RJ (Riester 1938) \\
\hline Terricola (Tricladida) & Pasipha chimbeva (E.M. Froehlich 1955) & São Paulo/SP (E.M. Froehlich 1955a) \\
\hline Terricola (Tricladida) & Pasipha hauseri (Froehlich 1959) & $\begin{array}{l}\text { Belém/PA (Froehlich \& E.M. Froehlich 1972) } \\
\text { São Leopoldo/RS (Froehlich 1959) }\end{array}$ \\
\hline Terricola (Tricladida) & Pasipha oliverioi (Froehlich 1955) & Teresópolis/RJ (Froehlich 1955b) \\
\hline Terricola (Tricladida) & Pasipha pasipha (Marcus 1951) & São Paulo/SP (Marcus 1951) \\
\hline Terricola (Tricladida) & Pasipha penhana (Riester 1938) & Penha/PB (Riester 1938) \\
\hline Terricola (Tricladida) & Pasipha pinima (E.M. Froehlich 1955) & São Paulo/SP (E.M. Froehlich 1955a) \\
\hline Terricola (Tricladida) & Pasipha plana (Schirch 1929) & Teresópolis/RJ (E.M. Froehlich 1955a) \\
\hline Terricola (Tricladida) & Pasipha pulchella (Schultze \& Müller 1857) & Blumenau/SC (Froehlich 1955b) \\
\hline Terricola (Tricladida) & Pasipha rosea (E.M. Froehlich 1955) & $\begin{array}{l}\text { Pirassununga/SP (E.M. Froehlich 1955a) } \\
\text { São Paulo/SP (E.M. Froehlich 1955a) } \\
\text { São Simão/SP (Froehlich 1958) } \\
\text { Lapa/PR (Froehlich 1956b) }\end{array}$ \\
\hline Terricola (Tricladida) & Pasipha splendida (Graff 1899) & Teresópolis/RJ (Graff 1899) \\
\hline Terricola (Tricladida) & Pasipha tapetilla (Marcus 1951) & $\begin{array}{l}\text { Rio de Janeiro/RJ (Froehlich 1956a) } \\
\text { Teresópolis/RJ (Froehlich 1956a) } \\
\text { Pirassununga/SP (Marcus 1951) } \\
\text { Ubatuba/SP (Froehlich 1956a) } \\
\text { Blumenau/SC (Froehlich 1959) } \\
\text { Itajaí/SC (Froehlich 1959) }\end{array}$ \\
\hline Terricola (Tricladida) & Pasipha trina (Marcus 1951) & Santo André/SP (Marcus 1951) \\
\hline Terricola (Tricladida) & Pasipha velina (Froehlich 1955) & $\begin{array}{l}\text { Blumenau/SC (Froehlich 1959) } \\
\text { Brusque/SC (du Bois-Reymond Marcus 1951b) }\end{array}$ \\
\hline Terricola (Tricladida) & Pasipha velutina (Riester 1938) & Nova Lima/MG (Riester 1938) \\
\hline
\end{tabular}


Tabela 2. Continuação...

\begin{tabular}{|c|c|c|}
\hline Ordem & Espécie & Município/Estado \\
\hline Terricola (Tricladida) & Pseudogeoplana bonita (Schirch 1929) & Teresópolis/RJ (Schirch 1929) \\
\hline Terricola (Tricladida) & Pseudogeoplana brasiliensis (Blainville 1826) & $\begin{array}{l}\text { Rio de Janeiro/RJ (Graff 1899) } \\
\text { Santos/SP (Graff 1899) }\end{array}$ \\
\hline Terricola (Tricladida) & Pseudogeoplana burri (Riester 1938) & Teresópolis/RJ (Riester 1938) \\
\hline Terricola (Tricladida) & Pseudogeoplana cardosi (Schirch 1929) & Teresópolis/RJ (Schirch 1929) \\
\hline Terricola (Tricladida) & Pseudogeoplana collini (Graff 1899) & RS* (Graff 1899) \\
\hline Terricola (Tricladida) & Pseudogeoplana doederleini (Schirch 1929) & Rio Doce*/RJ (Schirch 1929) \\
\hline Terricola (Tricladida) & Pseudogeoplana flava (Moseley 1877) & BA* (Moseley 1877) \\
\hline Terricola (Tricladida) & Pseudogeoplana goeldii (Graff 1899) & Rio de Janeiro/RJ (Graff 1899) \\
\hline Terricola (Tricladida) & Pseudogeoplana lumbricoides (Schirch 1929) & Teresópolis/RJ (Schirch 1929) \\
\hline Terricola (Tricladida) & Pseudogeoplana marginata (Schultze \& Müller 1857) & Blumenau/SC (Schultze \& Müller 1857) \\
\hline Terricola (Tricladida) & $\begin{array}{l}\text { Pseudogeoplana maximiliani (Schultze \& Müller } \\
\text { 1857) }\end{array}$ & Blumenau/SC (Schultze \& Müller 1857) \\
\hline Terricola (Tricladida) & Pseudogeoplana nephelis (Schultze \& Müller 1857) & Blumenau/SC (Schultze \& Müller 1857) \\
\hline Terricola (Tricladida) & Pseudogeoplana olivacea (Schultze \& Müller 1857) & Blumenau/SC (Schultze \& Müller 1857) \\
\hline Terricola (Tricladida) & $\begin{array}{l}\text { Pseudogeoplana pauloschirchi Ogren \& Kawakatsu } \\
1992\end{array}$ & Teresópolis/RJ (Schirch 1929) \\
\hline Terricola (Tricladida) & Pseudogeoplana pavonina (Riester 1938) & Teresópolis/RJ (Riester 1938) \\
\hline Terricola (Tricladida) & Pseudogeoplana perspicillata (Graff 1899) & Blumenau/SC (Graff 1899) \\
\hline Terricola (Tricladida) & Pseudogeoplana riedeli (Schirch 1929) & Rio de Janeiro/RJ (Riester 1938) \\
\hline Terricola (Tricladida) & Pseudogeoplana rostrata (Graff 1899) & Blumenau/SC (Graff 1899) \\
\hline Terricola (Tricladida) & Pseudogeoplana schirchi Ogren \& Kawakatsu 1990 & Teresópolis/RJ (Schirch 1929) \\
\hline Terricola (Tricladida) & Pseudogeoplana theresopolitana (Schirch 1929) & Teresópolis/RJ (Schirch 1929) \\
\hline Terricola (Tricladida) & Pseudogeoplana tricolor (Riester 1938) & Teresópolis/RJ (Riester 1938) \\
\hline Terricola (Tricladida) & Pseudogeoplana tristriata (Schultze \& Müller 1857) & Blumenau/SC (Schultze \& Müller 1857) \\
\hline Terricola (Tricladida) & Pseudogeoplana wetzeli (Schirch 1929) & Rio Doce* perto de Maylasky (Schirch 1929) \\
\hline Terricola (Tricladida) & Rhynchodemus blainvillei Graff 1899 & Blumenau/SC (Graff 1899) \\
\hline Terricola (Tricladida) & Rhynchodemus pellucidus Graff 1899 & $\begin{array}{l}\text { Santo André/SP (Marcus 1952) } \\
\text { Blumenau/SC (Graff 1899) }\end{array}$ \\
\hline Terricola (Tricladida) & Rhynchodemus piptus Marcus 1952 & São Paulo/SP (Marcus 1952) \\
\hline Terricola (Tricladida) & $\begin{array}{l}\text { Rhynchodemus schubarti du Bois-Reymond Marcus } \\
1955\end{array}$ & Pirassununga/SP (du Bois-Reymond Marcus 1955b) \\
\hline Terricola (Tricladida) & Rhynchodemus scius du Bois-Reymond Marcus 1955 & $\begin{array}{l}\text { Serra do Navio/AP (Froehlich \& E.M. Froehlich } \\
\text { 1972) } \\
\text { Porto Grande/AP (Froehlich \& E.M. Froehlich 1972) } \\
\text { Ubatuba/SP (du Bois-Reymond-Marcus 1955b) } \\
\text { Pirassununga/SP (du Bois-Reymond-Marcus 1955b) }\end{array}$ \\
\hline Terricola (Tricladida) & Rhynchodemus? hectori Graff 1897 & Teresópolis/RJ (du Bois-Reymond Marcus 1955b) \\
\hline Terricola (Tricladida) & Supramontana irritata Carbayo \& Leal-Zanchet 2003 & $\begin{array}{l}\text { São Francisco de Paula/RS } \\
\text { (Carbayo \& Leal-Zanchet 2003) }\end{array}$ \\
\hline Terricola (Tricladida) & Xerapoa hystrix Froehlich 1955 & $\begin{array}{l}\text { Mogi das Cruzes/SP (Froehlich 1955a) } \\
\text { São Paulo/SP (Froehlich 1955a) } \\
\text { Curitiba/PR (Froehlich 1955a) }\end{array}$ \\
\hline Terricola (Tricladida) & Xerapoa una Froehlich 1955 & Brusque/SC (Froehlich 1955a) \\
\hline
\end{tabular}

enorme extensão das águas territoriais brasileiras, e a variedade de ambientes (costeiro, estuarino, recifal e ambientes mais profundos), pode se esperar que coletas nesses ambientes, ainda não amostrados, revelem numerosas espécies novas.

Tricladida, Cavernicola. No Brasil nunca foram pesquisados ambientes de cavernas com o objetivo de coletar especificamente turbelários. Nas raras vezes em que foram coletados tricladidos, com uma única exceção, os animais eram imaturos. Os espécimes maduros, constituindo esta exceção, foram identificados (Kawakatsu \& E. M. Froehlich 1992) como Girardia paramensis (Fuhrman 1914). Trata-se de uma espécie troglófila de Paludicola. A única espécie brasileira de Cavernicola, Rhodax evelinae Marcus 1946, é de ambientes abertos. Até pouco tempo conhecida apenas do estado de São Paulo, tem sido recentemente encontrada no Rio Grande do 
Tabela 3. Números absoluto e relativo de gêneros e espécies de macroturbelários ocorrentes no Brasil e total de gêneros conhecidos no mundo

Table 3. Absolute and relative numbers of genera and species of Brazilian macroturbellarians, and number of known genera in the world.

\begin{tabular}{lcccccc}
\hline & Polycladida & Maricola & Cavernicola & Paludicola & Terricola & Total \\
\hline Espécies no mundo & 931 & 95 & 5 & 426 & 830 & 2287 \\
Espécies no Brasil (\% mundial) & $66(7,1)$ & $7(7,4)$ & $1(20,0)$ & $12(2,8)$ & $160(19,3)$ & $246(10,7)$ \\
Gêneros no mundo & 155 & 38 & 4 & 60 & 53 & 310 \\
Gêneros no Brasil (\% mundial) & $37(23,9)$ & $7(18,4)$ & $1(25,0)$ & $2(3,3)$ & $17(32,1)$ & $64(20,6)$ \\
Gêneros endêmicos (\% mundial) & $7(4,5)$ & $3(7,9)$ & $1(25,0)$ & $1(1,7)$ & $6(11,3)$ & $18(5,8)$ \\
\hline
\end{tabular}

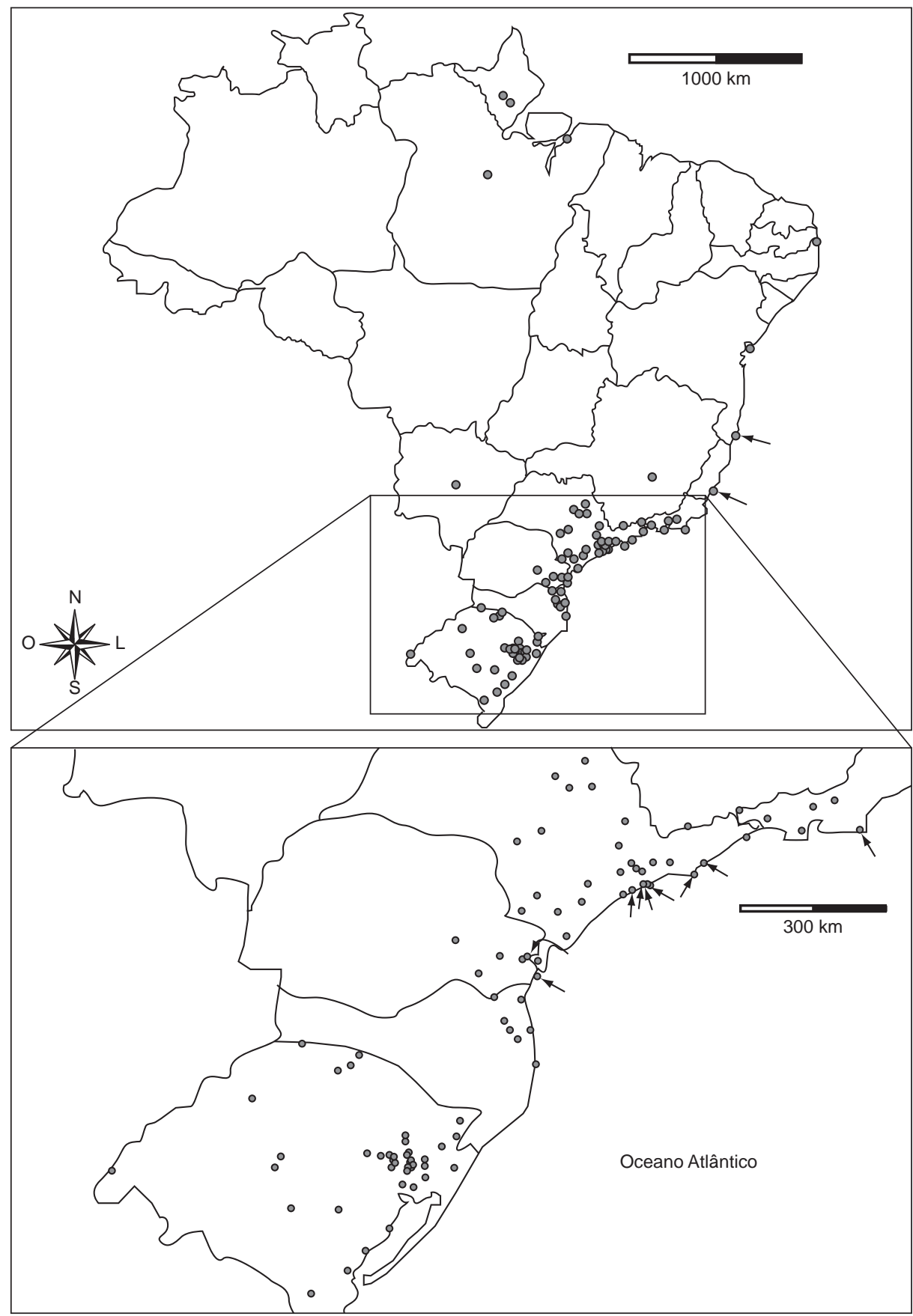

Figura 1. Municípios brasileiros com registros de Tricladida (círculos cinzas) e Polycladida (setas). A região meridional está ampliada para maior clareza. Figure 1. Brazilian municipalities with known Tricladida (gray circles) and Polycladida (arrows). For clarity purposes, the meridional region was amplified. 
Tabela 4. Municípios e registros (em parênteses) de macroturbelários, por região e por ambiente. Cada registro corresponde à citação de uma espécie em um município. Duas espécies (1,7\% do total) marinhas, cujo estado de procedência não é conhecido, foram contempladas no cómputo geral de espécies.

Table 4. Municipalities and records (in parentheses) of macroturbellarians, by region and environment. Each record refers to the citation of a species in a municipality. Two species (1.7\% of the total number), from marine environment, whose region of sampling is unknown, were added to the total number of species.

\begin{tabular}{|c|c|c|c|c|c|c|}
\hline Ambiente & Norte & Nordeste & Centro-Oeste & Sudeste & Sul & Total \\
\hline Límnico & $2(2)$ & $1(1)$ & $1(1)$ & $6(15)$ & 31 (47) & $43(66)$ \\
\hline Marinho & $0(0)$ & $1(2)$ & - & $9(82)$ & $2(4)$ & $14(90)$ \\
\hline Terrestre & $3(8)$ & $3(4)$ & $1(1)$ & 34 (205) & 36 (126) & 77 (344) \\
\hline Brasil & $5(10)$ & $5(7)$ & $2(2)$ & 45 (302) & 62 (177) & $121(500)$ \\
\hline Brasil \% & $4,1(2,0)$ & $4,1(1,4)$ & $1,7(0,4)$ & $37,2(60,4)$ & $51,2(35,4)$ & $100,0(100,0)$ \\
\hline
\end{tabular}

Sul, em arrozais, nos canais de irrigação (Vara \& Leal-Zanchet, com. pess.).

Tricladida, Paludicola. A quase totalidade das espécies brasileiras deste grupo é conhecida das regiões Sudeste e Sul (Figura 1), em razão do maior esforço amostral realizado nessas regiões (Sluys et al. 1997). Exceção notável é a da espécie acima referida, G. paramensis. Originalmente encontrada em um riacho nos Páramos colombianos, no Brasil foi coletada em cavernas na bacia do rio Xingu, no estado do Pará (Kawakatsu \& Froehlich 1992). Há ainda vastas regiões no país não amostradas, como indica a Figura 1. No Rio Grande do Sul há numerosos municípios amostrados, mas o número de espécies coletadas é pequeno quando comparado ao número dos terrícolos coletados nas mesmas regiões.

Tricladida, Terricola. Este grupo, o mais rico em espécies descritas, será ainda acrescido de várias outras, à medida que o estudo em andamento de várias espécies, coligidas por vários pesquisadores em diversas localidades, for sendo terminado. Resultados preliminares de parte desse material, coletado em matas do sul do país, revelaram de 13 a 40 espécies por localidade (Leal-Zanchet \& Carbayo 2000, Carbayo et al. 2002, de Castro \& Leal-Zanchet 2005, Fick et al. 2006, Baptista et al. 2006, Antunes et al. 2008). Porém, como nesses trabalhos muitos dos animais têm ainda o status de morfoespécie, não é possível saber qual o número de espécies novas e o grau de endemicidade nas matas pesquisadas.

Mesmo nas poucas localidades relativamente bem estudadas novas espécies podem ser encontradas. Por exemplo, apesar do grande esforço amostral realizado por E.M. e C.G. Froehlich e alunos, desde 1950, no início da construção da Cidade Universitária, no Butantan, São Paulo/SP, e em suas proximidades, ainda é possível encontrar pelo menos três espécies desconhecidas pela ciência (obs. pess.). Uma delas (descrição em fase de acabamento), ausente das primeiras coletas, e presente a partir dos anos 60, deve ter sido inadvertidamente introduzida com plantas ornamentais para os jardins (Carbayo et al. 2008).

\section{Atividade taxonômica atual}

Atualmente há três especialistas em taxonomia de Tricladida vinculados a núcleos de pesquisa no Brasil. Isso representa $0,65 \%$ dos 542 zoólogos taxonomistas brasileiros com vínculo estável (Marques \& Lamas 2006). Nos últimos cinco anos estes pesquisadores descreveram seis espécies e propuseram dois gêneros de Terricola. Não há sistematas de Polycladida. No contexto mundial, nos últimos cinco anos foram propostos 59 novos táxons (48 espécies, 9 gêneros, 1 subfamília, 1 família), publicados em 25 trabalhos, 4 deles (16,0\%) de autores brasileiros. Os trabalhos foram assinados por um total de 39 autores oriundos de 16 países (Tabela 5). O Brasil - junto da Rússia e dos EUA - é um dos países com maior número de taxonomistas, e, ao lado da Austrália, o que tem o maior número de centros de pesquisa.
Tabela 5. Nacionalidade dos 39 autores que assinam os 25 artigos com novos táxons de macroturbelários publicados nos últimos cinco anos, e localização dos centros de pesquisa aos quais os autores estão vinculados.

Table 5. Nacionality of the 39 authors that signed the 25 papers dealing with new macroturbellarian taxa published in the last 5 years, with the location of the research institutions to which the authors are associated.

\begin{tabular}{lccc}
\hline \multicolumn{1}{c}{ País } & $\begin{array}{c}\text { Primeiro } \\
\text { autor }\end{array}$ & $\begin{array}{c}\text { Total de } \\
\text { autores }\end{array}$ & $\begin{array}{c}\text { Centros de } \\
\text { pesquisa }\end{array}$ \\
\hline Austrália & 4 & 7 & 3 \\
Brasil & 4 & 4 & 3 \\
Bélgica & 1 & 3 & 3 \\
Holanda & 5 & 3 & 2 \\
Rússia & 2 & 4 & 2 \\
EUA & 1 & 4 & 2 \\
Japão & 2 & 2 & 1 \\
Argentina & 2 & 1 & 1 \\
Reino Unido & 2 & 1 & 1 \\
Espanha & 1 & 3 & 1 \\
Itália & 1 & 2 & 1 \\
Alemanha & - & 1 & 1 \\
Coréia do Sul & - & 1 & 1 \\
Nova Zelândia & - & 1 & 1 \\
Uruguai & - & 1 & 1 \\
Zimbábue & - & 1 & 1 \\
$\quad$ Total & 25 & 39 & 25 \\
\hline
\end{tabular}

Os dados referentes à atividade taxonômica no contexto mundial estão super-estimados, uma vez que foram incluídos entre eles autores sem vínculo estável (graduandos ou pós-graduandos) e pesquisadores aposentados ou mortos. Os dados mostram a relativa vitalidade da atividade taxonômica no Brasil no que diz respeito aos macroturbelários, embora o número de especialistas seja ainda muito insuficiente para trabalhar a diversidade do grupo. A escassez de especialistas para determinar as espécies estende-se a muitos outros grupos de invertebrados, alguns mesmo sem especialistas, e até a alguns grupos de vertebrados (Marques \& Lamas 2006, Migotto \& Marques 2006, Lewinsohn 2006, Lewinsohn \& Prado 2004, Amorim et al. 2002). Esta também é a realidade para plantas não vasculares (Giulietti et al. 2005) e bactérias (Lambais et al. 2006). São eloqüentes os doze séculos que, segundo estimativas de Lewinsohn \& Prado (2006), seriam necessários para descrever toda a biodiversidade brasileira, se mantido o ritmo da atividade taxonômica do período 1978-1995 focalizado por eles. 
Dentre as possíveis explicações para a escassez de taxonomistas de macroturbelários podemos citar os hábitos crípticos desses animais, sua relativa raridade e o trabalhoso e demorado processamento histológico exigido para seu estudo (Winsor 2001, Sluys \& Kawakatsu 2005). Acrescente-se a isso, e de uma maneira geral para muitos outros táxons, o lento recrutamento de novos sistematas, em parte devido ao fascínio que outros ramos mais novos da Biologia têm exercido sobre os iniciantes. Rieger (1998), no longo artigo em que expõe, quase exaustivamente, a pesquisa multifacetada sobre os turbelários desenvolvida durante os últimos 100 anos, considera jovem ainda o estudo do grupo. E termina: "As phylogenetic studies show us how important turbellarians are in the broad scheme of animal evolution, they should be ever more interesting subjects for study".

\section{Agradecimentos}

Agradecemos a Fernando Portella de Luna Marques (USP) o auxílio para a confecção do mapa; a Ramon Arthur Clark pela revisão do inglês; a Adriano S. Melo (UFRGS), do corpo editorial, e a dois assessores anônimos, pelas valiosas sugestões que muito contribuíram para melhorar o artigo. FC tem o apoio da Fundación BBVA (Biocon 06/067) e da FAPESP (proc. 2007/03890-6).

\section{Referências Bibliográficas}

ALMEIDA, E.C.J.D., YAMADA, C.M. \& FROEHLICH, E.M. 1988. Estudos cromossômicos em duas espécies de planárias terrestres: Geoplana abundans Graff e Issoca rezendei (Schirch) (Platyhelminthes, Tricladida, Terricola). Ciênc. Cult. Supl. 40:764-765.

ALMEIDA, E.J.C.D., YAMADA, C.M. \& FROEHLICH, E.M. 1991 Cytogenetic studies of two land planarian species from Brazil: Geoplana marginata and Issoca rezendei (Tricladida, Terricola). Hydrobiologia 227(1):169-173.

ALVAREZ, L. \& ALMEIDA, E.J.C.D. 1999. Comparative karyotypic analysis of two land planarian species from Brazil: Geoplana burmeisteri and Geoplana carinata (Tricladida, Terricola). Hereditas 131(1):1-4.

ALVAREZ, L. \& ALMEIDA, E.J.C.D. 2002. The first record of polyploidy in a land planarian species (Geoplana burmeisteri). Caryologia 55(3):235-239

ALVAREZ, L. \& ALMEIDA, E.J.C.D. 2007. Comparative karyotype analysis in diploid and triploid Dolichoplana carvalhoi (Tricladida, Terricola, Rhynchodemidae) from Brazil. Genet. Mol. Biol. 30(2):375-379.

AMORIM, D.S., SILVA, V.C. \& BALBI, M.I.P.A. 2002. Estado do conhecimento dos Diptera neotropicais. In Proyecto de Red Iberoamericana de Biogeografia y Entomología Sistemática (C. Costa, S.A. Vanin, J.M. Lobo, A. Melic, orgs.). Sociedad Entomológica Aragonesa y CYTED, Zaragoza, p. 29-36.

ANÔNIMO. 1987. Uma nova planária na Antártica. Comissão Interministerial para Recursos do Mar. Informativo 2(3):3.

ANTUNES, M.B., MARQUES, D.I.L. \& LEAL-ZANCHET, A.M. 2008. Composição das comunidades de planárias terrestres (Platyhelminthes, Tricladida, Terricola) em duas áreas de oresta estacional semidecidual do sul do Brasil. Neotrop. Biol. Conserv. 3(1):34-38.

BALL, I. 1969. An annotated checklist of the freshwater Tricladida of the Nearctic and Neotropical Regions. Can. J. Zool. 47:59-64.

BALL, I. 1971. Systematic and biogeographical relationships of some Dugesia species (Tricladia, Paludicola) from Central and South America. Am. Mus. Novitates 2472:1-25.

BAPTISTA, V.A., MATOS, L.B., FICK, I.A. \& LEAL-ZANCHET, A.M. 2006. Composição das comunidades de planárias terrestres (Platyhelminthes, Tricladida, Terricola) do Parque Nacional dos Aparados da Serra, Brasil. Iheringia, Sér. Zool. 96(3):293-297.

BAPTISTA, V.D.A., LEAL-ZANCHET, A.M. 2005. Nova espécie de Geoplana Stimpson (Platyhelminthes, Tricladida, Terricola) do sul do Brasil. Rev. Bras. Zool. 22(4):875-882.
BARROS, G.S., ANGELIS, D.F., FURLAN, L.T. \& CORRÊA-JUNIOR, B. 2006. Utilização de planárias da espécie Dugesia (Girardia) tigrina em testes de toxicidade de e uente de refinaria de petróleo. J. Braz. Soc. Ecotoxicol. 1(1):67-70

BELL, F.J. 1900. Remarks on a collection of land planarians made by Dr. Goeldi in Brazil. P. Zool. Soc. London 266-267.

BENYA, E., LEAL-ZANCHET, A.M., SANTOS, W.H., HAUSER, J. \& ERDTMANN, B. 2007. Chromosome polymorphism and complements in populations of Girardia species (Platyhelminthes, Tricladida, Paludicola) from southern Brazil. Braz. J. Biol. = Rev. Bras. Biol. 67(4):951-955.

BLAINVILLE, D.D. 1826. Planaires. In Dictionnaire Des Sciences Naturelles. Paris, p. 215-216.

BÖHMIG, L.V. 1887. Planaria iheringii, eine neue Triclade aus Brasilien. Zool. Anz. 10:482-483.

BORELLI, A. 1898. Viaggio del Dr. Enrico Festa nell'Ecuador e regione vicine. IX. Planarie d'acqua dolce. Boll. Mus. Zool. Anat. Comp. 13(322):1-6.

BUENO-SILVA, M. \& FISCHER, M.L. 2005A. Estudo da biologia de Girardia tigrina (Girard, 1850) (Platyhelminthes: Tricladida: Paludicola): análise do crescimento, reprodução e mortalidade em laboratório. Acta Biol. Leopoldensia 27(1):11-17.

BUENO-SILVA, M. \& FISCHER, M.L. 2005b. Dinâmica populacional e fenologia de Girardia tigrina (Girard, 1850) (Platyhelminthes: Paludicola) no Parque Barigüi, Curitiba - PR. Acta Biol. Leopoldensia 27(2):93-98.

BUENO-SILVA, M. \& FISCHER, M.L. 2007. Ocelos supernumerários espontâneos em Girardia tigrina (Platyhelminthes, Paludicola) no Estado do Paraná. Biociências 14(2):168-173.

CAMPOS-VELHO, N.M.R.D., LOPES, K.A.R. \& HAUSER, J. 2004. Morphometry of the eyes in regenerant of genus Dugesia (Platyhelminthes, Turbellaria, Dugesiidae). Braz. J. Biol. = Rev. Bras. Biol. 64(1): 1-9.

CARBAYO, F. \& LEAL-ZANCHET, A.M. 2001. A new species of terrestrial planarian (Platyhelminthes: Tricladida: Terricola) from South Brazil. Braz. J. Biol. $=$ Rev. Bras. Biol. 61(3):437-447.

CARBAYO, F. \& LEAL-ZANCHET, A.M. 2003. Two new genera of Geoplaninae (Terricola: Tricladida: Platyhelminthes) of Brazil in the light of cephalic apomorphies. Invert. Syst. 17(3):449-468.

CARBAYO, F. 2005. Procedimentos de campo e laboratório para a caracterização das planárias terrestres neotropicais (Platyhelminthes: Tricladida). Caderno La Salle XI 2(1):131-144.

CARBAYO, F. 2006. Redescription of two land planarian species of Notogynaphallia Ogren \&. Kawakatsu (Platyhelminthes, Tricladida, Geoplaninae) and confirmation of the heterogeneity of the genus. Braz. J. Zool. 23(3):746-757.

CARBAYO, F., LEAL-ZANCHET, A.M. \& VIEIRA, E.M. 2002. Terrestrial atworm (Platyhelminthes: Tricladida: Terricola) diversity vs. maninduced disturbance in a subtropical rainforest from Southern Brazil. Biodiversity Conserv. 11:1091-1104.

CARBAYO, F., LEAL-ZANCHET, A.M., VIEIRA, E.M. 2001. Land planarians (Platyhelminthes, Tricladida, Terricola) as indicators of man-induced disturbance in a south Brazilian rainforest. Belg. J. Zool. 131(Suppl. 1):223-224.

CARBAYO, F., PEDRONI, J. \& FROEHLICH, E.M. 2008. Colonization and extinction of land planarians (Platyhelminthes, Tricladida) in a Brazilian Atlantic Forest regrowth remnant. Biol Invasions 10(7):1131-1134.

CARLÉ, R. 1935. Beiträge zur Embriologie der Landplanarien. Ztschr. Morphol. Ökol. Tiere 29(4):527-558.

CARRANZA, S., LITTLEWOOD, D.T.J., CLOUG, K.A., RUIZ-TRILLO, I., BAGUÑÀ, J. \& RIUTORT, M. 1998. A robust molecular phylogeny of the Tricladida (Platyhelminthes: Seriata) with a discussion on morphological synapomorphies. Proc. R. Soc. Lond. B. 265: 631-640.

CARVALHO, I.M.D., BRUNNER, JR.A. \& VALENTE, M.M. 1984a. Relationship between the dorsoventral muscle fibers and the epidermis of Geoplana carinata Riester, 1938 (Platyhelminthes, Turbellaria, Tricladia). Zool. Jb. Anat. 111:25-33. 
CARVALHO, I.M.D., HAUSER, J. \& FRIEDRICH, M.G. 1975. Lichtmikroskopische Präparationsmethoden zur Darstellung der Basalmembran bei Geoplana carinata Riester (Turbellaria, Tricladida). Mikroskopie 31:323-332.

CARVALHO, I.M.D., VALENTE, M.M. KÖHLER, F.W. 1984b. Die Feinstruktur der Basalmembran bei Geoplana carinata Riester, 1938 (Platyhelminthes - Turbellaria). Z. Mikrosk. - Anat. 98(3):403-416.

CASTRO, R.D.C. \& LEAL-ZANCHET, A.M. 2005. Composição de comunidades de planárias terrestres (Platyhelminthes) em áreas de oresta estacional decidual e de campo na Região Central do Rio Grande do Sul, Brasil. Acta Biol. Leopoldensia. 27(3):147-150.

CORRÊA, D.D. 1947. A primeira Dolichoplana (Tricladida, Terricola) do Brasil. Bol. Fac. Fil. Ci. Letras, Univ. São Paulo, Zool. 12:57-81.

CORRÊA, D.D. 1949. Sobre o gênero Zygantroplana. Bol. Fac. Fil. Ciênc., Sér. Zool. 99:173-218.

CORREAA, D.D. 1958. A new polyclad from Brazil. Bol. Inst. Oceanogr. São Paulo 7:81-86.

CORRÊA, D.D. 1964. Turbellaria marinhos. In História natural de organismos aquáticos do Brasil. Fapesp, São Paulo, p. 101-102.

DARWIN, C. 1844. Brief descriptions of several terrestrial planariae, and of some remarkable marine species, with an accopunt of their habits. Quart. Jour. Micros. Soc. 91(2):209-214.

DIESING, K.M. 1861. Revision der Turbellarien. Abtheilung: Dendrocoelen. Sitzungsberichte d. mathemat.-Naturwiss. Classe der kaiserl. Akad. Wiss. 44 I Abt. 6-10:485-578

DU BOIS-REYMOND MARCUS, E. \& MARCUS, E. 1968. Polycladida from Curação and faunistically related regions. Stud. Fauna Curação 26:1-106.

DU BOIS-REYMOND MARCUS, E. 1951a. Contributions to the natural history of Brazilian Turbellaria. Comun. Zool. Mus. Hist. Nat. Montevideo $3(63): 1-25$.

DU BOIS-REYMOND MARCUS, E. 1951b. On South American geoplanids. Bol. Fac. Fil. Ci. Letras, Univ. São Paulo, Zool. 16:217-255.

DU BOIS-REYMOND MARCUS, E. 1955a. Chave dos Polycladida do litoral de São Paulo. Bol. Fac. Fil. Ci. Letras, Univ. São Paulo, Zool. 19:281-288.

DU BOIS-REYMOND MARCUS, E. 1955b. On Turbellaria and Polygordius from the Brazilian coast. Bol. Fac. Fil. Ci. Letras, Univ. São Paulo, Zool. 20:19-53.

DU BOIS-REYMOND MARCUS, E. 1957. On Turbellaria. Acad. Brasil. Ciênc. 29(1):153-191.

DU BOIS-REYMOND MARCUS, E. 1958. On South American Turbellaria. Acad. Brasil. Ciênc. 30(3):391-417

DU BOIS-REYMOND MARCUS, E. 1965. Drei neotropische Turbellarien. Sitz. - Ber. Ges. naturf. Freunde, Berlin 5:129-135.

EHLERS, U. 1985. Das Phylogenetische System der Plathelminthes. Gustav Fischer, Stuttgart, New York, 317 p.

FALLENI, A., LUCCHESI, P., GHEZZANI, C., SILVEIRA, M. \& GREMIGNI, V. 2006. Ultrastructural and cytochemical aspects of the female gonad of Geoplana burmeisteri (Platyhelminthes, Tricladida, Terricola). Jour. Morphol. 267(3):318-332.

FAUBEL, A. 1983. The Polycladida, Turbellaria. Proposal and establishment of a new system. Part I. The Acotylea. Mitt. Hamb. Zool. Mus. Inst. $80: 17-121$.

FAUBEL, A. 1984. The Polycladida, Turbellaria. Proposal and establishment of a new system. Part II. The Cotylea. Mitt. Hamb. Zool. Mus. Inst. 80:189-259.

FERNANDES, M.C., ALVARES, E.P., GAMA, P. \& SILVEIRA, M. 2003. Serotonin in the nervous system of the head region of the land planarian Bipalium kewense. Tissue Cell 35(6):479-486.

FÉRUSSAC, A.E.D. 1821. Note sur une nouvelle Espèce de ver terrestre du Brésil. Journ. de Physique 92:233-235.

FICK, I.A., LEAL-ZANCHET, A.M. \& VIEIRA, E.M. 2006. Community structure of land atworms (Platyhelminthes, Terricola): comparisons between Araucaria and Atlantic forest in southern Brazil. Invert. Biol. 125(4):306-313.

FIORENTIN, G.L. \& FRIEDRICH, S.M.G. 1985. Contribuição para o estado histológico do integumento auricular de Dugesia schubarti (Marcus, 1946). Acta Biol. Leopoldensia 7(1):119-130.

FORNERIS, L. 1999. Platelmintos turbelários. In Joly, C.A., Bicudo, C.E.M., orgs. Biodiversidade do Estado de São Paulo, Brasil: síntese do conhecimento ao final do século XX. 4: Invertebrados de Água Doce / Deborah Ismael, Wagner Cotroni Valenti, Takako Matsumura-Tundisi, Odete Rocha. FAPESP, São Paulo.

FROEHLICH, C.G. 1955a. Sôbre morfologia e taxonomia das Geoplanidae. Bol. Fac. Fil. Ci. Letras, Univ. São Paulo, Zool. 19:195-279.

FROEHLICH, C.G. 1955b. Notas sôbre geoplanas brasileiras. Pap. Avul. Dep. Zool. 12(7):189-198.

FROEHLICH, C.G. 1955c. On the biology of land planarians. Bol. Fac. Fil. Ci. Letras, Univ. São Paulo, Zool. 20:263-271.

FROEHLICH, C.G. 1956a. Tricladida Terricola das regiões de Teresópolis e Ubatuba. Pap. Avul. Dep. Zool. 12(16):313-344.

FROEHLICH, C.G. 1956b. Planárias terrestres do Paraná. Dusenia 7(4):173-196.

FROEHLICH, C.G. 1958. On a collection of brazilian land planarians. Bol. Fac. Fil. Ci. Letras, Univ. São Paulo, Zool. 21:93-121.

FROEHLICH, C.G. 1959. On geoplanids from Brazil. Bol. Fac. Fil. Ci. Letras, Univ. São Paulo, Zool. 22:201-265.

FROEHLICH, C.G. 1964. Turbellaria límnicos. In História natural de organismos aquáticos do Brasil. Fapesp, São Paulo, p. 99-100.

FROEHLICH, C.G. 1966. Notas sobre a ecologia de planárias terrestres. In Progresos en biologia del suelo. Montevideo, Uruguay.

FROEHLICH, C.G. 1967. A contribution to the zoogeography of neotropical land planarians. Acta Zool. Lilloana 23:153-162.

FROEHLICH, E.M. \& FROEHLICH, C.G. 1972. Land planarians from the Amazonian Region. Pap. Avul. Dep. Zool. 26(2):29-45.

FROEHLICH, E.M. \& LEAL-ZANCHET, A.M. 2003. A new species of terrestrial planarian of the genus Notogynaphallia (Platyhelminthes, Tricladida, Terricola) from south Brazil and some comments on the genus. Rev. Bras. Zool. 20(4):745-753.

FROEHLICH, E.M. 1955a. Sôbre espécies brasileiras do gênero Geoplana. Bol. Fac. Fil. Ci. Letras, Univ. São Paulo, Zool. 19:289-369.

FROEHLICH, E.M. 1955b. Chave para a classificação das Geoplanas brasileiras. Pap. Av. Dep. Zool. 12(8):201-214.

FROEHLICH, E.M. 1985. Plathelminthes (Turbellaria). In Manual de técnicas para a preparação de coleções zoológicas. Sociedade brasileira de Zoologia, São Paulo.

GIULIETTI, A.M., HARLEY, R.M., QUEIROZ, L.P., WANDERLEY, M.G.L. \& VAN DEN BERG, C. 2005. Biodiversity and conservation of plants in Brazil. Conserv. Biol. 19(3):632-639.

GOELDI, E.A. 1894. Breve notícia acerca de alguns vermes interessantes do Brazil. Bol. Mus. Paraense Hist. Nat. Ethnogr. 1(1):39-44.

GRAFF, L.V. 1896. Über das System und die geographische Verbreitung der Landplanarien. Verhandl. d. Deutsch. Zool. Ges. 6:61-75.

GRAFF, L.V. 1899. Monographie der Turbellarien: II. Tricladida Terricola. Engelmann, Leipzig.

GUECHEVA, T., ERDTMANN, B., BENFATO, M.S. \& HENRIQUES, J.A. 2003. Stress protein response and catalase activity in freshwater planarian Dugesia (Girardia) schubarti exposed to copper. Ecotoxicol. Environ. Safety 56(3):351-357.

GUECHEVA, T., HENRIQUES, J.A. \& ERDTMANN, B. 2001. Genotoxic effects of copper sulphate in freshwater planarian in vivo, studied with single cell gel tt (comet assay). Mutat. Res. 497(1-2):19-27.

GUIMARÃES, J.P. \& HIRANO, K. 1971. The origin of regenerative cells in planarian. Rev. Bras. Pesquisas Méd. e Biol. 4(3):199-202. 
GUIMARÃES, J.P. \& HIRANO, K. 1973. Teratomorfogênese espontânea em colônia de planária (Dugesia tigrina). Rev. Bras. Pesquisas Méd. e Biol. 6(1-2): 43-50.

GUIMARÃES, J.P., KLACZKO, L.B., HIRANO, K., VAZ, E.M. \& MIGUEL, N.M.R. 1975. Re-expressão de antígenos embrionários em planárias em regeneração. Rev. Bras. Pesquisas Méd. Biol. 8(3-4):255-259.

HAUSER, J. \& FRIEDRICH, S.M.G. 1982. Morphogenesis of regenerating fragments of Dugesia schubarti (Turbellaria Tricladida). Exp. Cell Biol. 50:61-71.

HAUSER, J. \& HELLER, Z. 1983. Pigmentação de olhos regenerantes de Dugesia schubarti (Marcus, 1946) (Tricladida Paludicola). Acta Biol. Leopoldensia 8(2):305-312.

HAUSER, J. \& SANTOS, W.H. 1985a. Studies on regeneration in the Dugesia anderlani Kawakatsu et Hauser, 1983. Part I. Regeneration of the amputated head. Morphological study. Acta Biol. Leopoldensia 7(2):163-174.

HAUSER, J. \& SANTOS, W.H. 1985b. Studies on regeneration in the Dugesia anderlani Kawakatsu et Hauser, 1983. Part II. Regeneration of different parts. Acta Biol. Leopoldensia 7(2):197-216.

HAUSER, J. \& SANTOS, W.H. 1985c. Janus head is really possible! Acta Biol. Leopoldensia 7(2):225-244.

HAUSER, J. 1959a. Non-cellular regeneration process in the integument of the atworm Geoplana abundans. Oncology 25(3):258-268.

HAUSER, J. 1959b. Studien über die Bewegungen des Geoplana. Pesquisas (Zoologia) 19:1-23.

HAUSER, J. 1979. Modificações morfológicas nos olhos de Dugesia schubarti (Marcus, 1946) durante a formação da nova cabeça. Acta Biol. Leopoldensia 1(2):237-262.

HAUSER, J. 1985. Self-division: suicide or reproduction? Acta Biol. Leopoldensia 4(1):3-16.

HAUSER, J. 1987. Olhos irregulares em regenerantes autotomizados de Dugesia anderlani Kawakatsu et Hauser, 1983. Acta Biol. Leopoldensia 9(2):319-334.

HAUSER, J., DIAS, M.I. \& FRIEDRICH, S.M.G. 1980. Processos histomórficos na regeneração em uma cauda de Dugesia schubarti (Marcus, 1946). Acta Biol. Leopoldensia 2(2):183-196.

HAUSER, J., SILVEIRA, S.M.O.D. \& PAULA, C.D. 1979a. Morphometrische Arbeiten mit Hilfe der Lupenphotographie. Mikroskope 3:207-212.

HAUSER, J., SILVEIRA, S.M.O.D. \& CARVALHO, I.M.D. 1979c. Observations on the regeneration rhythm of a new head in different regions of the body of Dugesia schubarti. Acta Biol. Leopoldensia 1(2):225-236.

HAUSER, J., SILVEIRA, S.M.O.D. \& FRIEDRICH, S.M.G. 1979b. Études preliminaires sur les modifications morphologiques des regenerants de Dugesia schubarti (Marcus, 1946) (Turbellaria, Tricladida). Acta Biol. Leopoldensia, 1(1):5-15.

HELLER, Z. 1985a. Study of the type and morphological characters of the eyes of the Dugesia tigrina (Girard, 1850). Acta Biol. Leopoldensia 7(1):77-90.

HELLER, Z. 1985b. Study of the external morphology of the eyes of the Dugesia anderlani (Kawakatsu et Hauser, 1983). Acta Biol. Leopoldensia 7(1):109-118.

HENSEL, M.L.D. 1980. Dados morfométricos de quatro populações de Dugesia schubarti (Marcus, 1946). Acta Biol. Leopoldensia 2(2):167-174.

HYMAN, L.H. 1955. Miscellaneous marine and terrestrial atworms from South America. Am. Mus. Novitates 1742:1-33.

HYMAN, L.H. 1951. The invertebrates. Vol. 2. Platyhelminthes and Rhynchocoela: the Acoelomate Bilateria. McGraw Hill, New York.

IBGE 2001. http://www.ibge.com.br/servidor_arquivos_est/ (último acesso em 15/08/2008).

JUNG, Z.H., SILVA, I.C.D. \& CARDOSO, L.T. 1981. Estudo morfológico da transformação de cabeças amputadas em Dugesia schubarti (Marcus, 1946). Acta Biol. Leopoldensia 3(1):127-147.
KAWAKATSU, M. \& FROEHLICH, E.M.1992. Freshwater planarians from caves of Brazil: Dugesia paramensis (Fuhrmann, 1914) and Dugesia sp. (Turbellaria, Tricladida, Paludicola). J. Speleol. Soc. Japan 17:1-19.

KAWAKATSU, M. 1989. Morphological, karyological and taxonomic studies of freshwater planarians from south Brazil. IX. The final report. Occ. Pub. Biol. Lab. Fuji Women's College 21: 1-4.

KAWAKATSU, M., HAUSER, J. \& FRIEDRICH, S.M.G. 1976. The freshwater planaria from South Brazil. Bull. Natnl. Sci. Mus. Ser. A (Zoology) 2:205-223.

KAWAKATSU, M., HAUSER, J. \& FRIEDRICH, S.M.G. 1980. Morphological, karyological and taxonomic studies of freshwater planarians from South Brazil. I. A history of those studies and a list of localities in the vicinities of São Leopoldo. Bull. Fuji Women's Coll. Ser 2, 18:129-151.

KAWAKATSU, M., HAUSER, J. \& FRIEDRICH, S.M.G. 1983b. Morphological, karyological and taxonomic studies of freshwater planarians from South Brazil. V. Dugesia tigrina (Girard, 1850) from município Botucatu, Estado de São Paulo, and Dugesia schurbati (Marcus, 1946) from the vicinity of São Paulo. Bull. Fuji Women's Coll. 21:147-163.

KAWAKATSU, M., HAUSER, J. \& FRIEDRICH, S.M.G. 1985. Morphological, karyological and taxonomic studies of freshwater planarians from south Brazil. VII. Supplementary notes on Dugesia schubarti (Marcus, 1946) from the vicinity of São Leopoldo, Estado de Rio Grande do Sul (Turbellaria, Tricladida, Paludicola). Bull. Fuji Women's Coll., Ser II, 23:101-109.

KAWAKATSU, M., HAUSER, J. \& FRIEDRICH, S.M.G. 1986. Morphological, karyological and taxonomic studies of freshwater planarians from South Brazil. VIII. Four Dugesia species (D. tigrina, D. schubarti, D. anderlani, and D. ardnti) collected from several localities in Estado do Rio Grande do Sul (Turbellaria, Tricladida, Paludicola). Bull. Fuji Women's Coll. 24:41-62.

KAWAKATSU, M., HAUSER, J. \& PONCE DE LEÓN, R. 1992. Freshwater planarians from Uruguay and Rio Grande do Sul, Brazil: Dugesia ururiograndeana sp. nov. and Dugesia tigrina (Girard, 1850) (Turbellaria, Tricladida, Paludicola). Bull. Biogeogr. Soc. Japan 47:33-50.

KAWAKATSU, M., HAUSER, J., FRIEDRICH, S.M.G. \& SOUZA LIMA, O.D. 1982. Morphological, karyological and taxonomic studies of freshwater planarians from South Brazil. III. Dugesia tigrina (Girard, 1850) and Dugesia schubarti (Marcus, 1946) from the vicinities of São Carlos, Estado de São Paulo (Turbellaria, Tricladida, Paludicola). Bull. Fuji Women's College, Ser. II, 20:73-90.

KAWAKATSU, M., HAUSER, J., FRIEDRICH, S.M.G. \& YAMAYOSHI, T 1983a. Morphological, karyological and taxonomic studies of freshwater planarians from South Brazil. IV. Dugesia anderlani sp. nov. (Turbellaria, Tricladida, Paludicola), a new species from São Leopoldo in Estado de Rio Grande do Sul. Annot. Zool. Japoneses 56(3):96-208.

KAWAKATSU, M., OKI, I., TAMURA, S., YAMAYOSHI, T., HAUSER, J. \& FRIEDRICH, S.M.G. 1984. Morphological, karyological and taxonomic studies of freshwater planarians from south Brazil. VI. Dugesia schubarti (Marcus, 1946) from the vicinity of São Leopoldo, Estado de Rio Grande do Sul. (Turbellaria, Tricladida, Paludicola). Bull. Fuji Women's College Ser. II, 22:45-62.

KAWAKATSU, M., OKI, I., TAMURA, S., YAMAYOSHI, T., HAUSER, J. \& FRIEDRICH, S.M.G. 1981. Morphological, karyological and taxonomic studies of freshwater planarians from South Brazil. II. Dugesia tigrina (Girard, 1850) (Turbellaria, Tricladida, Paludicola). Bull. Fuji Women's College Ser II, 19:113-136.

KENK, R. 1974. Index to the genera and species of the freshwater triclads (Turbellaria) of the world. Smithsonian Contr. Zool. 183:1-90.

KNAKIEVICZ, T. \& BUNSELMEYER, F.H. 2008. Evaluation of copper effects upon Girardia tigrina freshwater planarians based on a set of biomarkers. Chemosphere 71(3):419-428.

KNAKIEVICZ, T., LAU, A.H., PRÁ, D. \& ERDTMANN, B. 2007. Biogeography and karyotypes of freshwater planarians (Platyhelminthes, Tricladida, Paludicola) in southern Brazil. Zool. Sci. 24:123-129. 
KNAKIEVICZ, T., VIEIRA, S.M., ERDTMANN, B. \& FERREIRA, H.B. 2006. Reproductive modes and life cycles of freshwater planarians (Platyhelminthes, Tricladida, Paludicola) from southern Brazil. Invert. Biol. 125(3):212-221.

LACERDA, A.D.S., FREITAS, G.D.F.A., SOMMER-VINAGRE, A.P., FARIA, D.H., GÜNTZEL, J.B., SCHRÖDER, N.T. \& WIILLAND, E.D.F. 2005. Tolerância da planária de água doce Girardia schubarti (Platyhelminthes, Turbellaria, Tricladida) ao fungicida Dithane (M-45). Rev. Inic. Ci. Ulbra 4:55-65.

LAMBAIS, M.R., CROWLEY, D.E., CURY, J.C., BÜLL, R.C. \& RODRIGUES, R.R. 2006. Bacterial diversity in tree canopies of the Atlantic Forest. Science 312:1917.

LAU, A.H., KNAKIEVICZ, T., PRÁ, D. \& ERDTMANN, B. 2007. Freshwater planarians as novel organisms for genotoxicity testing: analysis of chromosome aberrations. Environ. Mol. Mutag. 48:475-482.

LEAL-ZANCHET, A.M. \& BAPTISTA, V.A. 2008. Planárias terrestres (Platyhelminthes: Tricladida: Terricola) em áreas de oresta com araucária no Rio Grande do Sul. In Fonseca, C.R.S., Souza, A.F., Dutra, T.L., Leal-Zanchet, A.M., Backes, A. \& Ganade, G.M.S. (eds.). Florestas com Araucária: Ecologia, Conservação e Desenvolvimento Sustentável. Holos, Ribeirão Preto. No prelo.

LEAL-ZANCHET, A.M. \& CARBAYO, F. 2000. Fauna de planárias terrestres da Floresta Nacional de São Francisco de Paula, RS: uma análise preliminar. Acta Biol. Leopoldensia 22(1):19-25.

LEAL-ZANCHET, A.M. \& CARBAYO, F. 2001. Two new species of Geoplanidae (Platyhelminthes, Tricladida, Terricola) from Brazil. $J$. Zool., Lond. 253:433-446.

LEAL-ZANCHET, A.M. \& FROEHLICH, E.M. 2001. A species complex in the genus Notogynaphallia (Tricladida: Terricola). Belg. J. Zool. 131(suppl. 1):225-226.

LEAL-ZANCHET, A.M. \& FROEHLICH, E.M. 2006. A species complex in the genus Notogynaphallia Ogren and Kawakatsu (Platyhelminthes: Tricladida: Terricola) with a taxonomic revision of homonyms of Geoplana marginata Schultze \& Müller and a reinterpretation of Notogynaphallia caissara (Froehlich) anatomy. Belg. J. Zool. 136(1):81-100.

LEAL-ZANCHET, A.M. \& HAUSER, J. 1999. Penis glands of the dugesiid planarian Girardia schubarti (Platyhelminthes, Tricladida, Paludicola). Invert. Biol. 118(1):35-41.

LEAL-ZANCHET, A.M. \& SOUZA, S.A.D. 2003. Redescrição de Choeradoplana iheringi Graff (Platyhelminthes, Tricladida, Terricola). Rev. Bras. Zool. 20(3):523-530.

LEUCK, E. \& LUZ, P.L. 1980. Vergleichende Studien der Geschwindigkeit der Fortbewegung von Dugesia schubarti (Marcus, 1946) bei intakten und amputierten Tieren. Acta Biol. Leopoldensia 2(2):197-204.

LEWINSOHN, T.M. \& PRADO, P.I. 2004. Biodiversidade brasileira: síntese do estado atual do conhecimento. Contexto, São Paulo.

LEWINSOHN, T.M. (Org.). 2006. Avaliação do estado do conhecimento da diversidade biológica brasileira. Ministério do Meio Ambiente, Brasília, $269 \mathrm{p}$

LIMA, O.D.S. 1981. Toxidez do inseticida endrin para Dugesia tigrina (Turbellaria, Tricladida). Ciênc. Cultura 3(33):392-394.

LIMA, O.D.S. 1982. Efeitos do inseticida organoclorado Endrin sobre a regeneração de planária (Dugesia tigrina). Nota preliminar. Ciênc. Cultura 2(34):219-222.

LIMA, O.D.S. 1984. Ação do herbicida trifuralina sobre Dugesia tigrina (Turbellaria Tricladida). Ciênc. Cultura 36(11):1940-1945.

MAIDA, M. \& FERREIRA, B.P. 1997. Coral reefs of Brazil: an overview. In Proceedings of the 8th International Coral Reef Symposium (H.A. Lessios and I.G. Macintyre, eds.). Smithsonian Tropical Research Institute, Panamá, p. 263-274.

MARCUS, E. 1946. Sobre Turbellaria brasileiros. Bol. Fac. Fil. Ci. Letras, Univ. São Paulo, Zool. 11:5-253.

MARCUS, E. 1947. Turbelários marinhos do Brasil. Bol. Fac. Fil. Ci. Letras, Univ. São Paulo, Zool. 12:99-215.

MARCUS, E. 1948. Turbellaria do Brasil. Bol. Fac. Fil. Ci. Letras, Univ. São Paulo, Zool. 13:113-243.
MARCUS, E. 1949. Turbellaria brasileiros (7). Bol. Fac. Fil. Ci. Letras, Univ. São Paulo, Zool. 14:7-155.

MARCUS, E. 1950. Turbellaria brasileiros (8). Bol. Fac. Fil. Ci. Letras, Univ. São Paulo, Zool. 15:5-191.

MARCUS, E. 1951. Turbellaria brasileiros (9). Bol. Fac. Fil. Ci. Letras, Univ. São Paulo, Zool. 16:5-215.

MARCUS, E. 1952. Turbellaria brasileiros (10). Bol. Fac. Fil. Ci. Letras, Univ. São Paulo, Zool. 17:5-187.

MARCUS, E. 1954. Turbellaria Brasileiros - XI. Pap. Avulsos Dep. Zool. Secr. Agric. 24:419-489.

MARCUS, E. 1963. Eine neue Meerestriklade von Sao Paulo. Zool. Beitr. (N.S.) 9:441-446.

MARQUES, A.C. \& LAMAS, C.J.E. 2006. Taxonomia zoológica no Brasil: estado da arte, expectativas e sugestões de ações futuras. Pap. Av. Zool. 46(13):139-174.

MARTINS, M.E.Q.P. 1970. Two new species of Dugesia (Tricladida Paludicola) from the State of São Paulo, Brazil. An. Acad. Brasil. Ciênc. 42:113-118.

MELO, A.S. \& ANDRADE, C.F. 2001. Differential predation of the planarian Dugesia tigrina on two mosquito species under laboratory conditions. $J$. Am. Mosquito Control Ass. 17(1):81-83.

MELO, A.S., MACEDO, C.C. \& ANDRADE, C.F.S.D. 1995. Eficiência de Dugesia tigrina (Girard) Turbellaria, Tricladida) como agente controlador de imaturos do mosquito Aedes albopictus (Skuse) em pneus armadilha. An. Soc. Entomol. Bras. 25(2):321-327.

MIGOTTO, A.E. \& MARQUES, A.C. 2006. Invertebrados marinhos. In Avaliação do estado do conhecimento biodiversidade brasileira (Lewinsohn, org.). 1 ed. Ministério do Meio Ambiente, Brasília, p. 149-202.

MOSELEY, H.N. 1877. Notes on the Structure of several forms of Land Planarians, with a description of two new genera, and several new species, and a list of all species at present known. Q. J. Micr. Sci. 17:274-292.

OGREN, R.E \& KAWAKATSU, M. 1998. American neartic and neotropical land planarian (Tricladida: Terricola) faunas. Pedobiologia 42(5-6):441-451.

OGREN, R.E. \& KAWAKATSU, M. 1990. Index to the species of the family Geoplanidae (Turbellaria, Tricladida, Terricola) Part I: Geoplaninae. Bull. Fuji Women's College, Ser. I, 28:79-166.

OGREN, R.E., KAWAKATSU, M. \& FROEHLICH, E.M. 1997. Additions and corrections of the previous land planarian indices of the world (Turbellaria, Seriata, Tricladida, Terricola) Addendum IV. Geographic locus index: Bipaliidae, Rhynchodemidae (Rhynchodeminae, Microplaninae), Geoplanidae (Geoplaninae, Caenoplaninae, Pelmatoplaninae). Bull. Fuji Womens'College, Ser. I 35:63-103.

PALMA, I.A.D. 1984. Relações de comportamento de planárias Dugesia tigrina Girard, 1850 (Turbellaria, Tricladida) a estimulações mecânicas e à fragmentação. Ciênc. Cultura 36(3):460-464.

PEREIRA, M.E.Q. 1970. Método para estudo de cromossomos em planárias. Ciênc. Cultura 22(3):211-212.

PRÁ, D., LAU, A.H., KNAKIEVICZ, T., CARNEIRO, F.R. \& ERDTMANN, B. 2005. Environmental genotoxicity assessment of an urban stream using freshwater planarians. Mutat. Res. 585:79-85.

PREZA, D.D.L. \& SMITH, D.H. 2001. Use of newborn Girardia tigrina (Girard, 1850) in acute toxicity tests. Ecotoxicol. Environ. Saf. $50(1): 1-3$

PRUDHOE, S. 1985. A monograph on Polyclad Turbellaria. Oxford University Press, London.

RIEGER, R. 1998. 100 years of research of 'Turbellaria'. Hydrobiologia 383(1-3):1-27.

RIESTER, A. 1938. Beiträge zur Geoplaniden-Fauna Brasiliens. Abhandl. senkenberg. naturf. Ges. 441:1-88.

RODRIGUES, S.D.A. \& FROEHLICH, E.M. 1998. Filo Platyhelminthes Classe Turbellaria. In Joly, C.A., Bicudo, C.E.M, orgs. Biodiversidade do Estado de São Paulo, Brasil: síntese do conhecimento ao final do século XX. 3: Invertebrados Marinhos. FAPESP, São Paulo.

SANTOS, W.H. \& HAUSER, J. 1984. Irregular formations in the Dugesia schubarti (Marcus, 1946) regenerating process. A contribution to the 
problem of the axial gradient theory. Acta Biol. Leopoldensia 6(2):231240.

SAWAYA, P. \& UNGARETI, M.D. 1948. In uência da temperatura sobre o consumo de oxigênio pelas planárias. Bol. Fac. Fil. Ci. Letras, Univ. São Paulo, Zool. 13:329-334.

SCHIRCH, P. 1929. Sobre as planárias terrestres do Brasil. Bol. Mus. Nat. Rio de Janeiro 5:27-38.

SCHULTZE, M. \& MÜLLER, F. 1857. Beiträge zur Kenntnis der Landplanarien, nach Mithheilungen des Dr. Fritz Müller in Brasilien und nach eigenen Untersuchungen von Dr. Max Schultze. Ab. Natur. Ges. Halle 4:61-74.

SCHULTZE, M. 1857. Contributions to the knowledge of the terrestrial planariae, from communications from Dr. Fritz Müller of Brazil and personal investigations. Am. Mag. Nat. Hist., Ser 2, 20:1-13.

SEITENFUS, A.L.R. \& LEAL-ZANCHET, A.M. 2004. Uma introdução à morfologia e taxonomia. de planárias terrestres. (Platyhelminthes, Tricladida, Terricola). Acta Biol. Leopoldensia 26(2):187-202.

SILVA, N.M.S., LEAL-ZANCHET, A.M. \& HAUSER, J. 1997. Analysis of the efficiency of different solutions for the fixation of Girardia tigrina (Turbellaria, Tricladida, Paludicola). Braz. J. Morphol. Sci. 14(2):271-274

SILVEIRA, M. \& CORINNA, A. 1976. Fine structural observations on the protonephridium of the terrestrial triclad Geoplana pasipha. Cell. Tiss. Res. 168(4):455-463.

SILVEIRA, M. 1969. Ultrastructural studies on a "Nine plus one" agellum, 1. J. Ultrastruct. Res. 26(3):274-288.

SILVEIRA, M. 1970. Characterization of an unusual nucleus by electron microscopy. Jour. Submicr. Cytol. 2:13-24.

SILVEIRA, M. 1973. Intraaxonemal glycogen in "9+1" agella of atworms. J. Ultrastr. Research 44(3):253-264.

SILVEIRA, M. 1974. The fine structure of 9+1 agella in Turbellarian atworms. In The Funcional anatomy of the spermatozoa (Afzelius, B.A., ed.). Pergamon Press, Oxford.

SILVEIRA, M. 1998. Ultrastructure of muscle cells from a few selected turbellarians: possible correlations between form and function. Hydrobiologia 383(1-3):191-196.

SLUYS, R. \& KAWAKATSU, M. 2005. Biodiversity of marine planarians revisited (Platyhelminthes, Tricladida, Maricola). J. Nat. Hist. 39(6):445-467.

SLUYS, R. 1989. A Monograph of the Marine Triclads. A.A. Balkema, Rotterdam

SLUYS, R. 1990. A monograph of the Dimarcusidae (Platyhelminthes, Seriata, Tricladida). Zool. Scr. 19(1):13-29.

SLUYS, R. 1996. Reconsiderations of species status of some South American planarians (Platyhelminthes: Tricladida: Paludicola). Proc. Biol. Soc. Washington 109(2):229-235.

SLUYS, R. 1999. Global diversity of land planarians (Platyhelminthes, Tricladida, Terricola): a new indicator-taxon in biodiversity and conservation studies. Biodiversity Conserv. 8(12):1663-1681.

SLUYS, R., HAUSER, J. \& WIRTH, Q.J. 1997. Deviation from the groundplan: a unique new species of freshwater planarian from south Brazil (Platyhelminthes, Tricladida, Paludicola). J. Zool., Lond. 241(3):593-601.

SLUYS, R., KAWAKATSU, M. \& PONCE DE LEÓN, R. 2005. Morphological stasis in an old and widespread group of species: Contribution to the taxonomy and biogeography of the genus Girardia (Platyhelminthes, Tricladida, paludicola). Stud. Neotrop. Fauna Environ. 40(2):155-180.

SMITH, E.H. 1960. On a new Polyclad commensal of Prosobranchs. An. Acad. Brasi. Ci. 32(3-4):1-4.

SOUZA, L.O.D. 1954. Preparação total de planárias e observações sobre a digestão. Ciênc. Cultura 6:162-164.

SOUZA, S.A.D. \& LEAL-ZANCHET, A.M. 2004. Histological and histochemical characterization of the secretory cells of Choeradoplana iheringi Graff, 1899 (Platyhelminthes: Tricladida: Terricola). Braz. J. Biol. = Rev. Bras. Biol. 64(3):511-522.

SOUZA, S.C., MUNIN, E., PROCOPIO ALVES, L., CASTILHO SALGADO, M.A., TAVARES PACHECO, M.T. 2005. Low power laser radiation at
$685 \mathrm{~nm}$ stimulates stem-cell proliferation rate in Dugesia tigrina during regeneration. J. Photochem. Photobiol., B 80(3):203-207.

SOUZA, S.T. \& LEAL-ZANCHET, A.M. 2002. Histological and histochemical aspects of the penial glands of Girardia biapertura Sluys, 1997 (Platyhelminthes, Tricladida, Paludicola). Braz. J. Biol. $=$ Rev. Bras. Biol. 2(3):547-555.

SOUZA, T.M.D. \& HAUSER, J. 1984. The growing of regenerating heads of Dugesia schubarti. Acta Biol. Leopoldensia 6(1):97-113.

SPALDING, M.D., FOX, H.E., ALLEN, G.R., DAVIDSON, N., FERDAÑA, Z.A., FINLAYSON, M., HALPERN, B.S., JORGE, M.A., LOMBANA, A., LOURIE, S.A., MARTIN, K.D., MCMANUS, E., MOLNAR, J., RECCHIA, C.A \& ROBERTSON, J. 2007. Marine ecoregions of the world: a bioregionalization of coastal and shelf areas. Bioscience 57(7):573-583

STEIGLEDER, A.K. \& HAUSER, J. 1984. Alimentação e crescimento de regenerantes de Dugesia schubarti (Marcus, 1946). Acta Biol. Leopoldensia 6(1):35-45.

TYLER, S., SCHILLING, S., HOOGE, M. \& BUSH, L.F. 2006. Turbellarian taxonomic database. Version $1.5 \mathrm{http}: / /$ turbellaria.umaine.ed (último acesso em 12/09/2007).

VALER, M.R. 2001. Observações sobre o crescimento populacional de planárias em condições laboratoriais: Girardia tigrina, Girardia schubarti e Girardia anderlani (Tricladida: Paludicola: Dugesiidae). Biociências 9(1):157-161.

VARA, D.C.D. \& LEAL-ZANCHET, A.M. 2008. Histological and histochemical characterization of secretory cells of the male copulatory organs of Girardia anderlani (Platyhelminthes: Tricladida: Paludicola). Braz. J. Zool. 25(2):263-268.

VARA, D.C.D., LEAL-ZANCHET, A.M. \& LIZARDO-DAUT, H.M. 2001. Histological processing techniques for the study of Dugesiidae development (Platyhelminthes, Tricladida, Paludicola). Rev. Bras. Biol. = Bras. J. Biol. 61(2):341-345.

VEYL, F.B.Z., GUEDES, H.L.M. \& SIMONE, S.G.D. 2002. Aspartic proteinase in Dugesia tigrina (Girard) planaria. Z. Naturforsch. Sect. C J. Biosci. 57(5-6):541-547.

WIILLAND, E.D.F., FRIEDRICH, S.M.G. \& HAUSER, J. 1998. Histologia do epitélio de Girardia schubarti (Marcus, 1946) (Platyhelminthes, Tricladida). 1. Divisão anatômica corporal. Acta Biol. Leopoldensia 20(2):319-327.

WIILLAND, E.D.F., HAUSER, J. \& FRIEDRICH, S.M.G. 2004. Histologia do tegumento de Girardia schubarti (Turbellaria, Tricladida, Paludicola). II. Tegumento. Biociências 12(2):89-98.

WINSOR, L. 2001. Confessions of a serial sectioner. Invertebrata: Tasmania's Inver. Newsletter, 20:12.

WIRTH, Q.J. \& HELLER, Z. 1985a. A in uência da alimentação, sangue de galinha, sôbre o crescimento e o desenvolvimento de Dugesia schubarti (Marcus, 1946) a partir da eclosão do casulo. Acta Biol. Leopoldensia 7(2):255-263.

WIRTH, Q.J. \& HELLER, Z. 1985b. In uência da alimentação com gema de ovo, cozida e crua, de galinha, no crescimento e desenvolvimento e na manutenção da vida de exemplares recém eclodido de Dugesia schubarti (Marcus, 1946). Acta Biol. Leopoldensia 7(2):265-274.

WIRTH, Q.J. \& HELLER, Z. 1985c. In uência da alimentação, proteína de soja e proteína de carne, no crescimento e desenvolvimento de exemplares recém eclodidos de Dugesia schubarti (Marcus, 1946). Acta Biol. Leopoldensia 7(2):275-284.

WIRTH, Q.J. \& HELLER, Z. 1985d. A in uência da alimentação, larva de mosquito, sôbre o crescimento, desenvolvimento e manutenção da vida e atingimento da maturidade sexual em exemplares recém eclodidos de Dugesia schubarti (Marcus, 1946). Acta Biol. Leopoldensia 7(2):285-291. 